OPEN ACCESS

Edited by:

Shawn Hayley,

Carleton University, Canada

Reviewed by:

Kah-Leong Lim,

National Neuroscience Institute,

Singapore

Dennis Qing Wang,

Third Affiliated Hospital of Sun

Yat-sen University, China

*Correspondence:

Kwang C. Chung

kchung@yonsei.ac.kr

Received: 03 November 2016 Accepted: 18 April 2017 Published: 11 May 2017

Citation:

Han KA, Yoo L, Sung JY, Chung SA, Um JW, Kim H, Seol W and

Chung KC (2017) Leucine-Rich Repeat Kinase 2 (LRRK2) Stimulates IL-1 $\beta$-Mediated Inflammatory Signaling through Phosphorylation of RCAN1.

Front. Cell. Neurosci. 11:125. doi: 10.3389/fncel.2017.00125

\section{Leucine-Rich Repeat Kinase 2 (LRRK2) Stimulates IL-1 $\beta$-Mediated Inflammatory Signaling through Phosphorylation of RCAN1}

\author{
Kyung A. Han ${ }^{1}$, Lang Yoo ${ }^{1}$, Jee Y. Sung ${ }^{2}$, Sun A. Chung ${ }^{3}$, Ji W. Um ${ }^{4}$, Hyeyoung Kim ${ }^{3}$, \\ Wongi Seol ${ }^{5}$ and Kwang C. Chung ${ }^{1 *}$ \\ ${ }^{1}$ Department of Systems Biology, College of Life Science and Biotechnology, Yonsei University, Seoul, South Korea, ${ }^{2}$ Center \\ for Pediatric Oncology, National Cancer Center, Goyang-si, South Korea, ${ }^{3}$ Department of Food and Nutrition, College of \\ Human Ecology, Yonsei University, Seoul, South Korea, ${ }^{4}$ Department of Brain and Cognitive Sciences, Daegu Gyeongbuk \\ Institute of Science and Technology (DGIST), Daegu, South Korea, ${ }^{5}$ InAm Neuroscience Research Center, Sanbon Medical \\ Center, College of Medicine, Wonkwang University, Gunpo-si, South Korea
}

Leucine-rich repeat kinase 2 (LRRK2) is a Ser/Thr kinase having mixed lineage kinase-like and GTPase domains, controlling neurite outgrowth and neuronal cell death. Evidence suggests that LRRK2 is involved in innate immune response signaling, but the underlying mechanism is yet unknown. A novel protein inhibitor of phosphatase $3 \mathrm{~B}, \mathrm{RCAN1}$, is known to positively regulate inflammatory signaling through modulation of several intracellular targets of interleukins in immune cells. In the present study, we report that LRRK2 phosphorylates RCAN1 (RCAN1-1S) and is markedly up-regulated during interleukin-1 $\beta$ (IL-1 $\beta$ ) treatment. During IL-1 $\beta$ treatment, LRRK2mediated phosphorylation of RCAN1 promoted the formation of protein complexes, including that between Tollip and RCAN1. LRRK2 decreased binding between Tollip and IRAK1, which was accompanied by increased formation of the IRAK1-TRAF6 complex. TAK1 activity was significantly enhanced by LRRK2. Furthermore, LRRK2 enhanced transcriptional activity of NF- $\mathrm{KB}$ and cytokine IL-8 production. These findings suggest that LRRK2 might be important in positively modulating $\mathrm{L}-1 \beta$-mediated signaling through selective phosphorylation of RCAN1.

\section{Keywords: LRRK2, RCAN1, interleukin-1 $\beta$, phosphorylation, NF-кB, Parkinson's disease}

\section{INTRODUCTION}

Parkinson's disease (PD) is the second-most common neurodegenerative disorder, and characterized by the formation of abnormal protein inclusions, referred as Lewy bodies (Moore et al., 2005). Numerous reports indicate that excessive inflammation in the brain is closely associated with the pathogenesis of and neural degeneration observed in PD. Many cytokines, including various interleukin (IL) subtypes and tumor necrosis factor $\alpha$ (TNF- $\alpha$ ), are elevated in the brains and cerebrospinal fluid of PD patients (Mogi et al., 1994, 1996). During the progression of $\mathrm{PD}$, neurotoxic molecules are released within neurons, which can trigger inflammatory signaling cascades.

Mutations in the gene encoding leucine-rich repeat kinase 2 (LRRK2) have also been linked to autosomal-dominant Parkinsonism (Li et al., 2014). Studies by others have demonstrated 
that LRRK2 is involved in the inflammatory response induced by $\alpha$-synuclein or lipopolysaccharide (LPS). For example, rats deficient in LRRK2 are protected from dopaminergic neurodegeneration caused by $\alpha$-synuclein over-expression or LPS exposure (Daher et al., 2014). Moreover, certain stimuli that induce inflammation are known to modulate the expression and activity of LRRK2. For example, interferon- $\gamma$ and LPS treatment enhance LRRK2 expression and activity (Gardet et al., 2010; Moehle et al., 2012; Kuss et al., 2014). Recent studies also suggest that LRRK2 can regulate two key regulators of innate immune response, nuclear factor of activated T-cells (NFAT) and nuclear

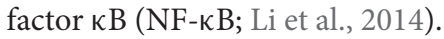

Interestingly, TNF- $\alpha$ expression and the resultant cell death in the microglial cells from LRRK2-R1441G transgenic mouse were increased by LPS stimulation (Gillardon et al., 2012). On the contrary, the expression of interleukin- $1 \beta$ and cyclooxygenase- 2 in microglial cells from LRRK2-knockout mouse was much reduced upon LPS treatment. The deletion of LRRK2 also promoted the phosphorylation of NF- $\kappa \mathrm{B}$-inhibitory subunit p50 at S337 and the nuclear accumulation of NF-кB (Russo et al., 2015).

IL-1 receptors (IL-1Rs) and Toll-like receptors (TLRs) both commonly contain an intracellular Toll/IL-1R (TIR) domain and serve as major receptors of innate immunity and inflammation (Martin and Wesche, 2002). IL-1 signaling is initiated by the ligand-induced formation of a receptor complex consisting of IL-1R and the IL-1R accessory protein. In this process, $\mathrm{MyD} 88$ recruitment appears to constitute the first step in a series of protein-protein interactions at activated receptor complexes (Martin and Wesche, 2002). Under unstimulated conditions, Toll-interacting protein (Tollip) interacts with IL-1R-associated kinase 1 (IRAK1), inhibiting downstream TLR signaling (Martin and Wesche, 2002). During stimulation with IL-1, MyD88IRAK1-Tollip complexes are recruited to the heterodimeric IL-1R. IRAK4 is simultaneously recruited to the receptor complex, phosphorylating IRAK1 and inducing IRAK1 autophosphorylation. Activated IRAK1 interacts with TNF receptorassociated factor 6 (TRAF6; Martin and Wesche, 2002). The TRAF6-IRAK1 complex dissociates from the receptor, and activated IRAK1 is subsequently degraded by the ubiquitin proteasome system (UPS). TRAF6 contains a RING domain and functions as an ubiquitin E3 ligase that conjugates Lys63-linked polyubiquitin chains to TRAF6 itself. Activated TRAF6 stimulates auto-phosphorylation of transforming growth factor- $\beta$-activated kinase 1 (TAK1), which then becomes fully activated. Regulatory kinases in downstream signaling pathways are phosphorylated by TAK1 (Martin and Wesche, 2002). Finally, NF- $\kappa \mathrm{B}$ and numerous inflammatory cytokines themselves become activated (Lawrence, 2009).

Regulator of calcineurin 1 (RCAN1; also known as DSCR1) inhibits calcium-dependent protein phosphatase 3 (calcineurin), consequently affecting many cellular responses, including lymphocyte activation and neuronal and muscle development (Park et al., 2009). There are four transcripts of RCAN1, but the major transcriptional products are isoforms which include exon 1 (RCAN1-1) or 4 (RCAN1-4; Park et al., 2009). Although RCAN1-1 consists of 197 amino acid (RCAN1-1S), an additional start site has been found upstream of exon 1, which produces a protein with 252 amino acids (RCAN1-1L; Park et al., 2009). RCAN1 regulates the activities of several inflammatory transcription factors. For example, RCAN1 acts as a negative modulator of calcineurin, leading to inhibition of NFAT activity (Fuentes et al., 2000; Rothermel et al., 2000; Vega et al., 2002). RCAN1 also affects NF- $\mathrm{B}$ activity and downstream cytokine signaling. For instance, RCAN1-1S interacts with Tollip, promoting its dissociation from the IRAK1 complex, which then stimulates NF- $\kappa B$ activity upon treatment with IL-1 $\beta$ (Lee et al., 2009).

Based on evidence suggesting a putative role of LRRK2 during inflammatory signaling, we investigated biochemical and functional interactions between LRRK2 and RCAN1 (specifically, RCAN1-1S), and a potential regulatory role for LRRK2 in RCAN1-mediated IL-1 $\beta$ inflammatory signaling. We determined RCAN1 to be a novel substrate of LRRK2, and that their interaction affects a key functional signalosome during IL-1 $\beta$-mediated inflammatory signaling.

\section{MATERIALS AND METHODS}

\section{Materials}

Peroxidase-conjugated anti-rabbit and anti-mouse antibodies were purchased from Millipore (Billerica, MA, USA). Dulbecco's modified Eagle's medium (DMEM), fetal bovine serum (FBS), fetal calf serum, and lipofectamine and PLUS reagents were purchased from Life Technologies (Grand Island, NY, USA). Anti-Myc, anti-GAPDH, anti-TAK1, anti-TRAF6 and anti-IRAK1 antibodies were purchased from Santa Cruz Biotechnology (Santa Cruz, CA, USA). Polyclonal anti-RCAN1 antibodies were purchased from ECM Biosciences (Versailles, KY, USA) and Santa Cruz Biotechnology. Anti-LRRK2, antiphospho-LRRK2 (Ser935), and anti-phospho-TAK1 (Thr187) antibodies were purchased from Abcam (Cambridge, MA, USA) and Cell Signaling Technology (Beverly, MA, USA), respectively. Polyclonal and monoclonal anti-HA antibodies were purchased from Abnova (Tebu, France) and Covance (Powhatan, VA, USA), respectively. Protein A-Sepharose beads and glutathione sepharose $4 \mathrm{~B}$ were purchased from GE Healthcare Life Science (Piscataway, NJ, USA). Enhanced chemiluminescence (ECL) reagent and $[\gamma-32 \mathrm{P}]$ ATP were purchased from Perkin Elmer Life Sciences (Downers Grove, IL, USA). MG132 was purchased from A.G. Scientific (San Diego, CA, USA). Human recombinant IL-1 $\beta$ was purchased from Sigma (St. Louis, MO, USA).

\section{cDNA Constructs}

Mammalian constructs encoding Myc-tagged wild-type LRRK2, its kinase-dead counterpart and two PD-associated pathogenic LRRK2 mutants (pcDNA3.1-Myc-LRRK2-WT, -D1994A, -G2019S and -R1441C, respectively) were generated, as described elsewhere (Shin et al., 2008; Heo et al., 2010). The mammalian expression vector for HA-tagged human wild-type RCAN1 (RCAN1-1S) was a kind gift from S. de la Luna (Genomics Regulation Center, Barcelona, Spain). Mammalian 
expression vectors encoding HA-tagged RCAN1 deletion mutants (HA-RCAN1 ${ }^{1-95}$, HA-RCAN1 ${ }^{1-125}$, HA-RCAN1 ${ }^{30-197}$, and HA-RCAN1 ${ }^{90-197}$ ) were constructed, as previously described (Lee et al., 2012). Bacterial expression vectors encoding GST-fused wild-type RCAN1 (pGEX4T-1-GSTRCAN1) and five deletion mutants (RCAN1 ${ }^{1-95}, \mathrm{RCAN}^{1-125}$, RCAN $1^{1-145}$, RCAN $1^{1-160}$ and RCAN $1^{1-175}$ ) were produced by PCR amplification of wild-type RCAN1 and its deletion mutants using Prime STAR-HS DNA Polymerase (TAKARA, Shiga, Japan) and sub-cloning into the pGEX4T-1 vector. Bacterial constructs encoding GST-fused RCAN1 mutants having a substitution at Ser149, Thr151, Thr152, Thr154, or Ser162 with alanine, respectively, were generated through PCR amplification of pGEX4T-1-GST-RCAN1-WT as a template. Site-directed mutagenesis reactions were performed using the Prime ${ }^{\circledR}$ STAR HS DNA polymerase (Takara Bio Inc., Shiga, Japan).

\section{Cell Culture and DNA Transfection}

LRRK2 +/+ and LRRK2 -/- mouse embryonic fibroblasts (MEFs) were kindly provided by D.R. Alessi (University of Dundee, Dundee, Scotland), and human embryonic kidney 293 (HEK293) cells stably expressing type 1 IL-1R (HEK293/IL-1RI) were a generous gift from G. Takaesu (Keio University, Tokyo, Japan). RAW264.7 cells, HEK293 cells, and LRRK2 $+/+$ and $-/-$ MEFs were cultured in DMEM containing 10\% FBS, $100 \mathrm{unit} / \mathrm{ml}$ penicillin, and $100 \mu \mathrm{g} / \mathrm{ml}$ streptomycin at $37^{\circ} \mathrm{C}$ in $5 \% \mathrm{CO}_{2}$. HEK293/IL-1RI cells were also cultured in DMEM containing $10 \%$ fetal calf serum, 100 unit $/ \mathrm{ml}$ penicillin, and $100 \mu \mathrm{g} / \mathrm{ml}$ streptomycin at $37^{\circ} \mathrm{C}$ in $5 \% \mathrm{CO}_{2}$. All DNA transfections were performed using the Lipofectamine and PLUS reagent according to the manufacturer's protocol.

\section{Immunoprecipitation and Western Blot Analysis}

Cells were rinsed with ice-cold phosphate-buffered saline (PBS), harvested in $1 \%$ Nonidet P40 lysis buffer $(50 \mathrm{mM}$ Tris, $\mathrm{pH} 7.5,1 \%$ Nonidet P40, $150 \mathrm{mM} \mathrm{NaCl}, 10 \%$ glycerol and protease inhibitor cocktail including $1 \mathrm{mM} \mathrm{Na}_{3} \mathrm{VO}_{4}, 1 \mu \mathrm{g} / \mathrm{ml}$ leupeptin, $1 \mu \mathrm{g} / \mathrm{ml}$ aprotinin, $10 \mathrm{mM} \mathrm{NaF}$ and $0.2 \mathrm{mM}$ phenylmethylsulfonyl fluoride), and briefly sonicated. Brain lysates were obtained from P28 mouse and brain tissues were lysed by RIPA buffer (50 mM Tris ( $\mathrm{pH} 7.4$ ), $150 \mathrm{mM} \mathrm{NaCl}$, $1 \%$ Triton X-100, 0.5\% sodium deoxycholate, $0.1 \%$ SDS, and protease inhibitor cocktail). All experimental protocols using mice were approved by the Institutional Animal Care and Use Committee of Yonsei University (the approval reference number: 2007-0004). Lysates were collected after centrifugation for $20 \mathrm{~min}$ at $4^{\circ} \mathrm{C}$. For immunoprecipitation, $1 \mu \mathrm{g}$ of the appropriate antibody was incubated overnight at $4^{\circ} \mathrm{C}$ with $0.8 \mathrm{mg}$ of cell extracts prepared in cell lysis buffer. The samples were incubated with $30 \mu \mathrm{l}$ of a 1:1 suspension of protein A-Sepharose beads for $2 \mathrm{~h}$ at $4^{\circ} \mathrm{C}$ with gentle rotation. Beads were pelleted by centrifugation at $10,000 \times g$ for $30 \mathrm{~s}$ at $4^{\circ} \mathrm{C}$, and washed three times with $1 \%$ Nonidet P40 lysis buffer. Immunocomplexes were dissociated by boiling in $2 \times$ SDS-PAGE sample buffer, separated by SDS-PAGE, and transferred to a nitrocellulose membrane. Membranes were then blocked in TBST buffer (20 mM Tris, pH 7.5, $137 \mathrm{mM}$ $\mathrm{NaCl}$, and $0.1 \%$ Tween 20 ) containing $5 \%$ nonfat dry milk for $1 \mathrm{~h}$ at room temperature, and incubated overnight at $4^{\circ} \mathrm{C}$ in TBST buffer containing 3\% nonfat dry milk and the appropriate primary antibody. Membranes were washed three times in TBST and incubated with the secondary IgG-coupled horseradish peroxidase antibody for $2 \mathrm{~h}$ at room temperature. Membranes were washed three times with TBST and the signal visualized using ECL reagent. Band intensities were measured using Multi Gauge v3.1 software (Fujifilm Life Science, Tokyo, Japan).

\section{Phos-Tag Immunoblotting}

After DNA transfection, cells were rinsed in ice-cold PBS, and then lysed with the lysis buffer containing $0.2 \%$ Nonidet P40, $50 \mathrm{mM}$ Tris ( $\mathrm{pH} 7.4$ ), $150 \mathrm{mM} \mathrm{NaCl}, 10 \%$ glycerol and protease inhibitor cocktail. Samples containing phosphorylated RCAN1 were separated by $10 \%$ SDS-PAGE containing $25 \mathrm{mM}$ Phos-tag (Wako Pure Chemical Industries, Osaka, Japan). Phos-tag immunoblotting was performed according to the manufacturer's protocol.

\section{GST Pull-Down Assay}

GST pull-down assays were performed by incubating GST or GST-fused RCAN1 immobilized onto GST beads for $2 \mathrm{~h}$ at $4^{\circ} \mathrm{C}$ with the cell lysates. The mixtures were washed three times with wash buffer $(25 \mathrm{mM}$ Tris- $\mathrm{HCl}, \mathrm{pH} 7.5,1 \mathrm{mM}$ dithiothreitol, $30 \mathrm{mM} \mathrm{MgCl}, 40 \mathrm{mM} \mathrm{NaCl}$, and $1 \%$ Nonidet P40). Bound proteins were eluted with $2 \times$ SDS buffer, separated by SDS/PAGE, and then subjected to Western blot analysis using an anti-Myc antibody.

\section{RNA Interference}

Small interference RNAs (siRNA) targeting human RCAN1 (siRNA no. 1044226) and scrambled siRNA (catalog no. SN-1013) were purchased from Bioneer (Daejeon, South Korea). HEK293/IL-1RI cells were transfected with $200 \mathrm{nM}$ siRNA using lipofectamine RNAiMAX (Life Technologies) according to the manufacturer's protocol.

\section{Luciferase Reporter Assay}

HEK293/IL-1RI and LRRK2 +/+ and LRRK2 -/MEF cells were mock-transfected or transfected with Myc-LRRK2 wild-type or kinase-dead constructs. NF-кBdependent firefly luciferase reporter and effector plasmids were co-transfected into the cells along with the Renilla luciferase plasmid. After $24 \mathrm{~h}$, cells were treated with IL-1 $\beta$ for $6 \mathrm{~h}$ and then harvested in a passive lysis buffer. Luciferase assays were performed using the Dual-Luciferase Reporter Assay System (Promega, Madison, WI, USA). Relative luciferase activity was calculated by dividing firefly luciferase activity by Renilla luciferase activity. Data represented three independent experiments performed in triplicate. 


\section{In Vitro Kinase Assay}

HEK293 cells were transfected for $24 \mathrm{~h}$ with plasmids encoding Myc-tagged wild-type LRRK2 or its kinase-dead mutant (LRRK2-D1994A). Cells were lysed in 1\% Nonidet P40 lysis buffer and cell lysates were immunoprecipitated overnight at $4^{\circ} \mathrm{C}$ with an anti-Myc antibody. The immunocomplexes were incubated with $30 \mu \mathrm{l}$ of a 1:1 protein A-Sepharose bead suspension for $2 \mathrm{~h}$ at $4^{\circ} \mathrm{C}$ with gentle inversion. Beads were centrifuged and washed twice with lysis buffer, and washed twice with $1 \times$ LRRK2 kinase reaction buffer (20 mM HEPES, pH 7.4, $15 \mathrm{mM} \mathrm{MgCl}_{2}, 5 \mathrm{mM}$ EGTA, 0.1\% Triton X-100, $0.5 \mathrm{mM}$ DTT, and $1 \mathrm{mM}$ glycerophosphate). The samples were then mixed with $2 \mu \mathrm{g}$ of bacterial recombinant GST-fused wild-type RCAN1 or its deletion mutants and $1 \times$ LRRK2 kinase reaction buffer containing $10 \mu \mathrm{M}$ cold-ATP. The in vitro kinase reaction was initiated by the addition of $5 \mu \mathrm{Ci}(\gamma-32 \mathrm{P}) \mathrm{ATP}$. The reaction was allowed to proceed for $15 \mathrm{~min}$ at $30^{\circ} \mathrm{C}$ before being terminated by the addition of SDS-PAGE sample buffer. Protein samples were resolved by SDS-PAGE and incorporated $(\gamma-32 \mathrm{P})$ radioisotope was detected by autoradiography.

\section{Real-Time PCR Analysis for IL-8 mRNA Expression}

Total RNAs were converted into cDNAs using Primescript RT master Mix kit (TAKARA). cDNA was incubated with SYBR Green Real-time PCR master mix (Toyobo Co. Ltd., Osaka, Japan) containing $10 \mathrm{pg} / \mathrm{ml}$ of the forward and reverse primers, and then amplified using the Light Cycler PCR system (Roche Applied Sciences, Indianapolis, IN, USA). Sequences of the IL-8 primers used were $5^{\prime}$-ATGACTTCCAAGCTGGCCGTGGCT-3' (forward) and $5^{\prime}$-TCTCAGCCCTCTTCAAAAACTTCT-3' (reverse), generating a 297-base pair PCR product. GAPDH was used as an internal control, the forward primer being $5^{\prime}$-GAAGGTGAAGGTCGGAGT- $3^{\prime}$ and the reverse primer being 5'-GAAGATGGTGATGGGATTC-3', generating a 226-base pair PCR product.

\section{Enzyme-Linked Immunosorbent Assay}

After DNA transfection, the amount of secreted IL-8 was determined using an ELISA kit (Biosource, Camarillo, CA, USA), according to the manufacturer's protocol.

\section{Statistical Analysis}

Data are represented as means \pm SEM. Group means were compared using Student's $t$-tests. $P$ values $<0.05$ were considered statistically significant.

\section{RESULTS}

\section{LRRK2 Physically Interacts with RCAN1-1S}

Both LRRK2 and RCAN1-1S regulate NF- KB transcriptional activity in the IL-1R/TLR signaling pathway (Lee et al., 2009; Li et al., 2014). Therefore, we sought to determine if there were any biochemical interactions between LRRK2 and RCAN1. We also investigated the potential functional and physiological significance, if any, of such an interaction, with particular focus on regulation of the IL-1 $\beta$-mediated inflammatory signaling pathway.

We first determined whether LRRK2 interacts with RCAN1 in mammalian cells using co-immunoprecipitation (co-IP) assays. After transfection of HEK293 cells with Myc-tagged LRRK2 and/or HA-tagged RCAN1 for $24 \mathrm{~h}$, cell lysates were immunoprecipitated using anti-HA or anti-Myc antibodies. Immunoblot analyses found that ectopically expressed LRRK2 binds to RCAN1 in HEK293 cells (Figures 1A,B). Co-immunoprecipitation analyses also demonstrated that endogenous LRRK2 binds to endogenous RCAN1, whereas there was no obvious interaction in immunocomplexes from samples prepared with IgG as a negative control (Figure 1C). We determined that LRRK2 interacts with RCAN1 in the mouse brain (Figure 1D). Furthermore, in vitro GST pull-down assays performed on HEK293 cell lysates with GST-fused RCAN1 demonstrated that LRRK2 directly binds RCAN1, whereas, this interaction was not observed in samples prepared with GST as a control (Figure 1E). Collectively, these data suggest that LRRK2 specifically interacts with RCAN1 in mammalian cells.

To determine which domain(s) of RCAN1 interact with LRRK2, deletion mutants of RCAN1 (RCAN1 $1^{1-95}$, RCAN1 ${ }^{1-125}$, RCAN1 $1^{30-197}$, and RCAN $1^{96-197}$ ) were generated and their ability to interact with LRRK2 was tested (Figure 2A). Co-IP assays found that wild-type LRRK2 binds several RCAN1 peptides, specifically, RCAN $1^{1-95}, \mathrm{RCAN}^{1-125}$, and RCAN1 $1^{30-197}$, as well as the full-length RCAN1 (Figures 2A,B). However, RCAN $1^{96-197}$ exhibited greatly diminished interaction with LRRK2 (Figures 2A,B). These results suggest that the $\mathrm{N}$-terminal 30-95 amino acid region of RCAN1 is necessary for LRRK2 binding.

\section{LRRK2 Directly Phosphorylates RCAN1}

The finding that LRRK2 interacts with RCAN1 raised the possibility that LRRK2, a protein kinase, can phosphorylate RCAN1. To test this hypothesis, in vitro kinase assays were performed using lysates from HEK293 cells transfected with Myc-tagged wild-type LRRK2 or a kinase-dead mutant (LRRK2-D1994A or LRRK2-KD) and bacterial recombinant GST-RCAN1 as a substrate. After incubation with anti-Myc immunocomplexes, GST-RCAN1, and $\left(\gamma_{-}{ }^{32} \mathrm{P}\right)$ ATP, autoradiography results showed that wild-type LRRK2, but not the LRRK2-KD mutant, directly phosphorylates RCAN1 in vitro (Figure 3A).

We further tested whether LRRK2 phosphorylates RCAN1 in transformed cell lines. HEK293 cells were transfected with HA-RCAN1 alone or with either Myc-tagged wild-type LRRK2 or Myc-LRRK2-KD. Analyses of cell lysates using phos-tag gel, which separates non-phosphorylated proteins from phosphorylated ones, depending on differential motility rates, showed that levels of phosphorylated RCAN1 were considerably higher in the presence of wild-type LRRK2, but not LRRK2-KD (Figure 3B). Two of the most common pathogenic PD-associated LRRK2 mutants, LRRK2-G2019S and LRRK2-R1441C, have been shown to have enhanced kinase activity. Both LRRK2-G2019S and LRRK2-R1441C increased 


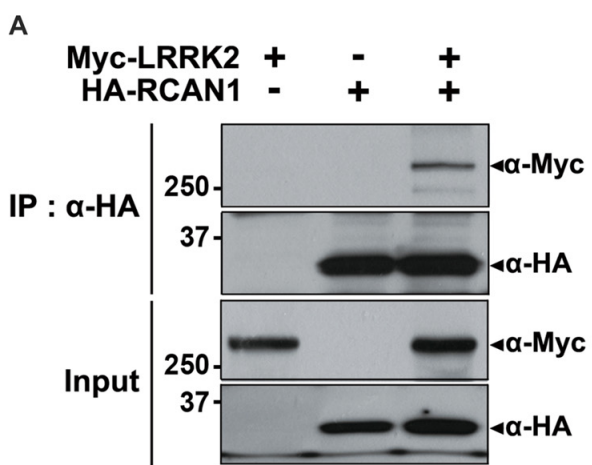

C

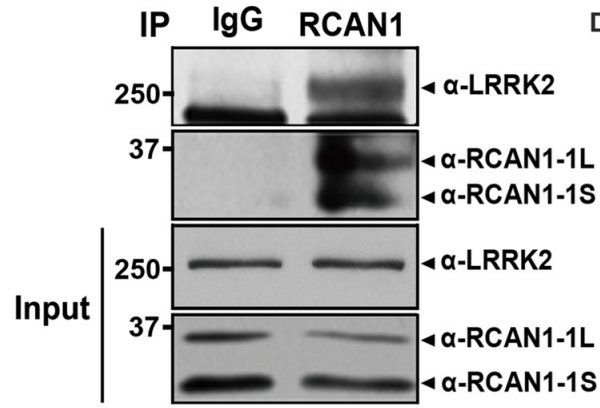

B

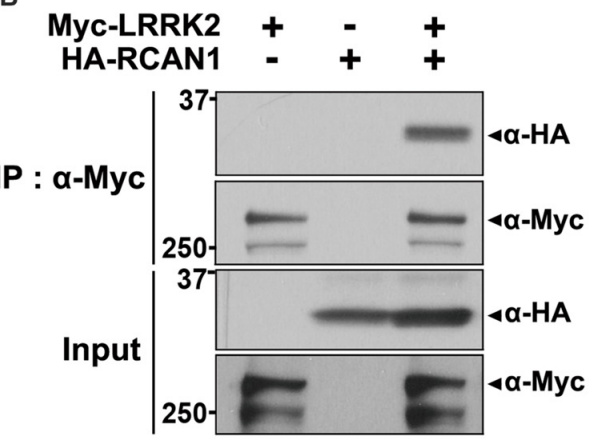

D

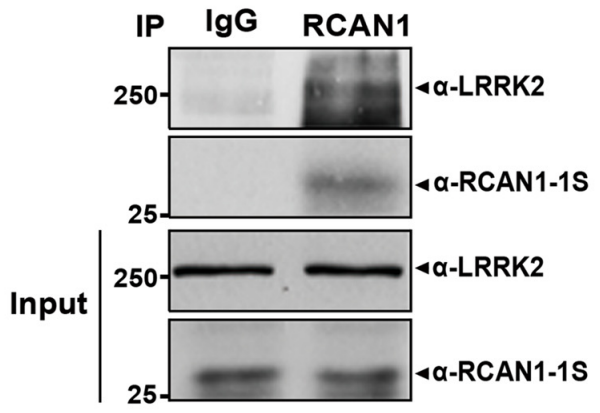

$\mathbf{E}$

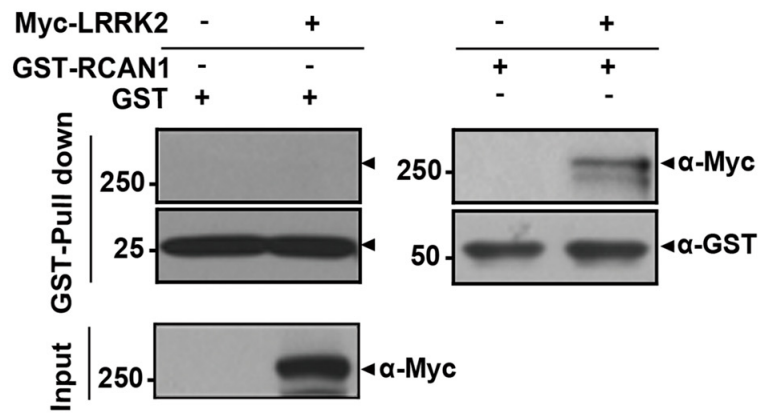

FIGURE 1 | Leucine-rich repeat kinase 2 (LRRK2) interacts with RCAN1. (A,B) Human embryonic kidney 293 (HEK293) cells were transfected for 24 h with plasmid encoding Myc-tagged wild-type LRRK2 and/or HA-tagged RCAN1. Total cell lysates were immunoprecipitated with anti-HA (A) or anti-Myc (B) antibodies, and immunocomplexes were analyzed using anti-Myc (A) or anti-HA (B) antibodies. Expression of proteins were determined using Western blotting with anti-Myc or anti-HA IgGs. (C) HEK293/IL-1RI cell lysates were immunoprecipitated with an anti-RCAN1 antibody, followed by immunoblotting with an anti-LRRK2 antibody. As a negative control, cell lysates were immunoprecipitated with pre-immune lgG (lgG), as indicated. Expression of LRRK2 and RCAN1 (RCAN1-1S and RCAN1-1L) in cell extracts was determined using immunoblotting with anti-LRRK2 or anti-RCAN1 antibodies. (D) Immunoprecipitation of mouse brain lysates was performed with an anti-RCAN1 antibody, followed by immunoblotting with an anti-LRRK2 antibody. As a control, cell lysates were immunoprecipitated with pre-immune lgG (IgG). Expression of LRRK2 and RCAN1-1S in tissue lysates was determined using immunoblotting with anti-LRRK2 or anti-RCAN1 antibodies. (E) GST pull-down assays were performed by incubating cell lysates prepared after DNA transfection with Myc-LRRK2-WT or empty vector for 24 h with GST-RCAN1-immobilized glutathione-sepharose. Bound complexes were analyzed using Western blotting with an anti-Myc antibody; GST served as a negative control. Purification of GST-fused RCAN1 and the presence of Myc-LRRK2 in cell extracts were confirmed using Western blotting with anti-GST or anti-Myc antibodies, respectively.

RCAN1 phosphorylation by $\sim 1.6$ and $\sim 1.3$-fold, respectively (Figure 3C).

Previous reports indicate that RCAN1 and LRRK2 are closely related to IL-1R/TLR signaling (Lee et al., 2009; Li et al., 2014). Therefore, we next addressed whether treatment with IL-1 $\beta$ affects or accelerates RCAN1 phosphorylation by LRRK2. To test this hypothesis, HEK293/IL-1RI cells stably expressing the type I IL- $1 \beta$ receptor, which exhibits a robust response to IL-1 $\beta$ stimulation (Cao et al., 1996), were utilized. After transfection with Myc-tagged LRRK2 for $24 \mathrm{~h}$, HEK293/IL-1RI cells were treated with $10 \mathrm{ng} / \mathrm{ml} \mathrm{IL-1 \beta}$ and anti-Myc immunocomplexes were isolated. In vitro kinase assays followed by autoradiography showed that stimulation with IL-1 $\beta$ promoted LRRK2 auto-phosphorylation and LRRK2-mediated RCAN1 phosphorylation (Figure 3D). These results indicate that treatment with IL-1 $\beta$ stimulates 


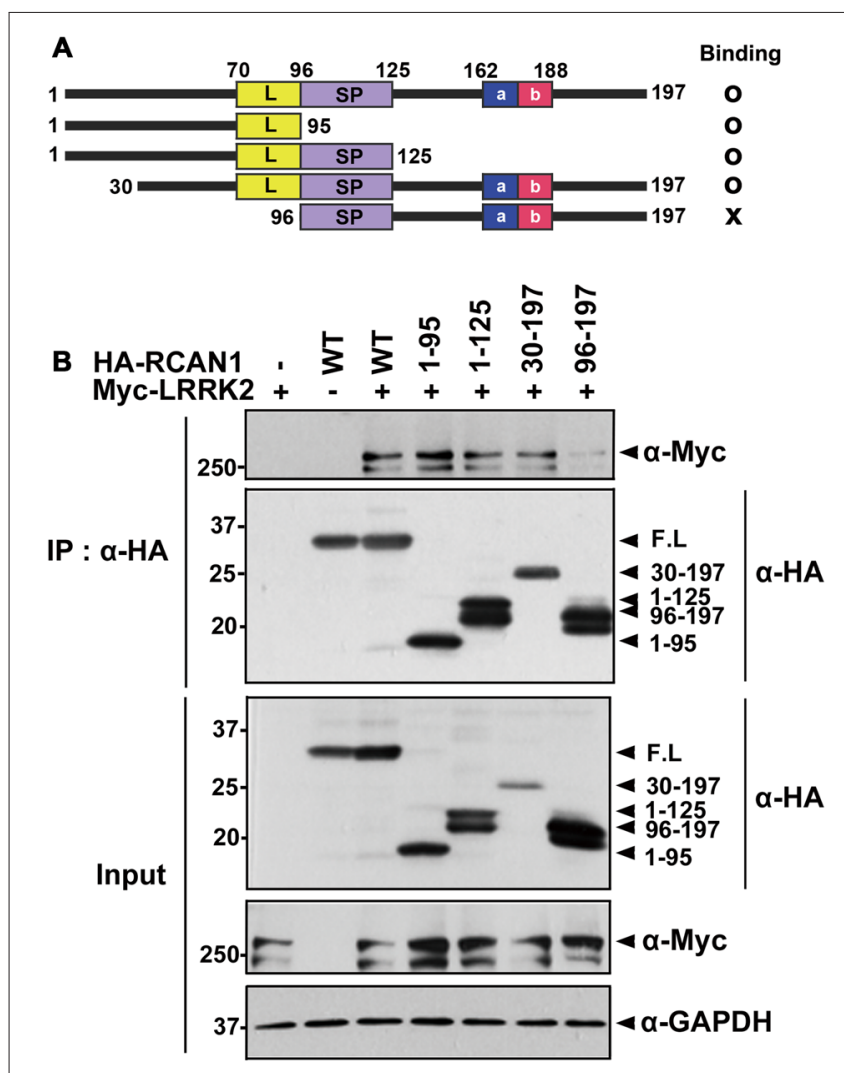

FIGURE 2 | The N-terminal 30-95 region of RCAN1 is important for binding with LRRK2. (A) Diagram of HA-tagged wild-type RCAN1-1S, its deletion mutants, and a summary of the binding assay results. RCAN1-1S consists of an N-terminal amphipathic leucine repeat $(\mathrm{L})$ domain, a central span of 31 amino acids containing a serine-proline (SP) repeat, a C-terminal acidic region (a), and a cluster of basic amino acids (b). (B) HEK293 cells were transfected for $24 \mathrm{~h}$ with Myc-LRRK2 alone or in combination with various HA-tagged deletion RCAN1 mutants, as indicated. Cell lysates were immunoprecipitated using anti-HA antibody. Immunocomplexes were analyzed using Western blotting with anti-Myc antibody, as indicated. Expression of Myc-LRRK2 and HA-RCAN1 was determined using Western blotting with anti-Myc or anti-HA antibodies. GAPDH served as a loading control.

RCAN1 phosphorylation by LRRK2 through an IL-1R-mediated signaling pathway.

To identify which region(s) in RCAN1 is important for LRRK2-mediated phosphorylation, in vitro kinase assays were performed using truncated GST-fused RCAN1 mutants. HEK293 cells were mock-transfected or transfected with Myc-tagged LRRK2, and cell lysates were immunoprecipitated with an anti-Myc antibody. In vitro kinase assays using antiMyc-LRRK2 immunocomplexes and recombinant GST-fused RCAN1 deletion mutants demonstrated that RCAN1 ${ }^{1-95}$, RCAN $1^{1-125}$ and RCAN1 $1^{1-145}$ were not phosphorylated by LRRK2 (Figures 3E-G), whereas RCAN1 ${ }^{1-175}$ and RCAN1 ${ }^{1-160}$ are phosphorylated weakly or much less, respectively (Figure 3G). These results indicated that the C-terminal 145-160 or 145-175 amino acid region of RCAN1 contains target(s) for LRRK2-mediated phosphorylation. As the 160-175 region contains only one Ser/Thr residue (Ser-162), we further examined whether it is directly phosphorylated by LRRK2. In vitro kinase assays by using the GST-fused RCAN1 mutant having a substitution at Ser162 with alanine (GST-RCAN1-S162A mutant) as a substrate revealed that this fragment is still phosphorylated by LRRK2 (Figure $\mathbf{3 H}$ ). As the 145-160 region contains four additional Ser/Thr residues for the phosphorylation (i.e., Ser149, Thr151, Thr152 and Thr154), we then generated four RCAN1 mutants having a substitution of each site with alanine, respectively. When we assessed the effect of LRRK2-mediated phosphorylation on these mutations, unexpectedly, all these point-mutants were still and substantially phosphorylated by LRRK2 (Figure 3I). These results indicate that LRRK2-targeted residue is not simply limited to the region spanning amino acids 145-175. These results also suggest that LRRK2-mediated phosphorylation of deletion mutants failed to reflect the phosphorylation pattern of wild-type RCAN1.

Next, we examined the phosphorylation efficiency of LRRK2 toward RCAN1 through comparison with RAB5B, another well-known target of LRRK2 (Yun et al., 2015). As shown in Figure 3J, in vitro kinase assays demonstrated that LRRK2 phosphorylated GST-RCAN1 more efficiently than RAB5B, confirming that RCAN1 is a novel substrate of LRRK2 kinase.

\section{LRRK2 Increases Association between RCAN1-1S and Tollip}

A previous report has found that RCAN1-1S interacts with Tollip and promotes IL-1 $\beta$-mediated inflammatory signaling (Lee et al., 2009). Consistent with this finding, our present results show that IL-1 $\beta$ treatment potentiates LRRK2-mediated phosphorylation of RCAN1. Taken together, these observations suggest that LRRK2 can modulate IL-1R-mediated downstream signaling.

To investigate a putative regulatory role for LRRK2 in inflammatory response, we assessed whether LRRK2 modulates interaction between RCAN1 and Tollip in response to IL-1 $\beta$ treatment. HEK293/IL-1RI cells were transfected with HA-RCAN1 and Xpress-Tollip alone or with LRRK2-WT or LRRK2-KD. Co-immunoprecipitation assays showed that there was increased interaction between RCAN1 and Tollip in the presence of LRRK2-WT, compared with control cells. However, RCAN1 binding to Tollip was reduced by LRRK2-KD (Figure 4A). To further verify the role of native LRRK2 in RCAN1-Tollip interaction, we tested the effect of GSK2578215A (GSK), a potent and highly selective inhibitor of LRRK2 kinase, on their binding. As a control, pretreatment of cells with GSK at $1 \mu \mathrm{M}$ for $5 \mathrm{~h}$ sufficiently inhibits the LRRK2 activity, as measured by LRRK2 phosphorylation at Ser935 (Deng et al., 2011; Figure 4B). As shown in Figure 4C, GSK treatment attenuated the interaction between RCAN1 and Tollip. In addition, the increased RCAN1 binding to Tollip in the presence of LRRK2 was much enhanced and sustained longer after IL-1 $\beta$ treatment (Figure 4D).

We then investigated whether two pathogenic LRRK2 mutations affect association between RCAN1 and Tollip. We evaluated binding affinity between RCAN1 and Tollip 
A Myc-LRRK2 - WT KD

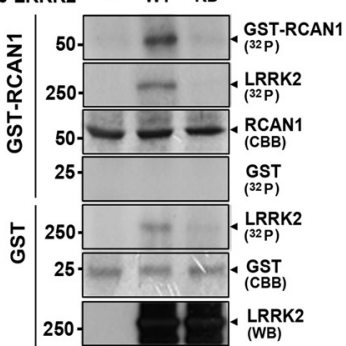

D

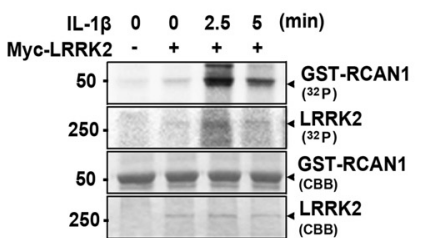

B

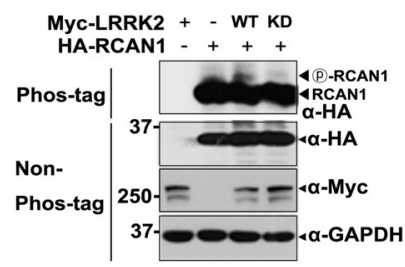

C
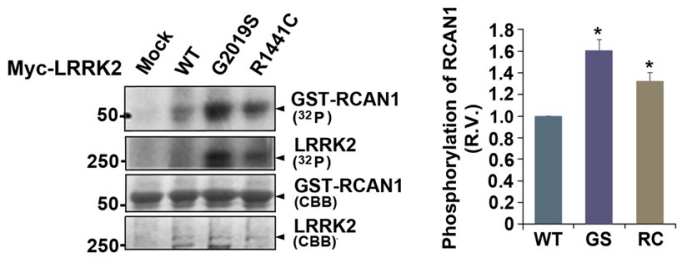

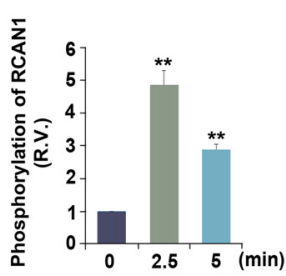

E

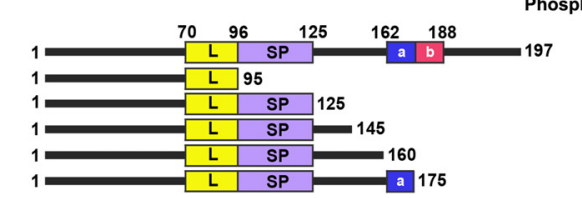

Phosphorylation
$\mathbf{F}$

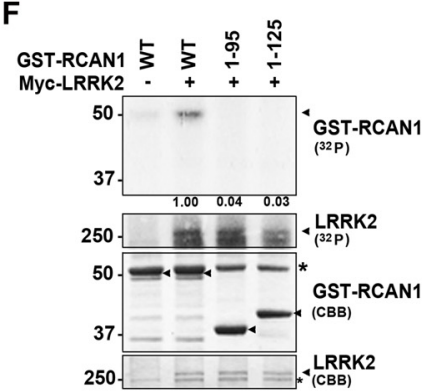

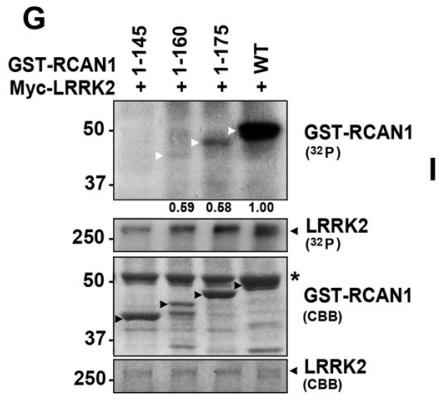

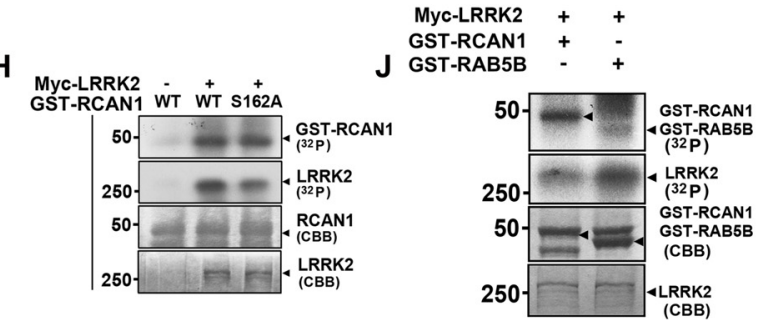

GST-RCAN1 WT WT S199P,151 T152 T15AA Myc-LRRK2

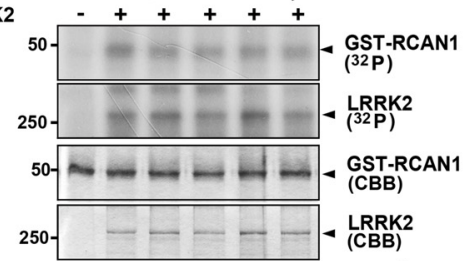

FIGURE 3 | LRRK2 directly phosphorylates RCAN1, which is enhanced by IL-1 $\beta$ treatment. (A) HEK293 cells were mock-transfected or transfected for $24 \mathrm{~h}$ with Myc-tagged wild-type LRRK2 (LRRK2-WT) or its kinase-dead mutant having the point mutation of D1994A (LRRK2-KD). Cell lysates were immunoprecipitated with an anti-Myc antibody, and in vitro kinase assay was performed. (B) HEK293 cells were transfected for $24 \mathrm{~h}$ with HA-RCAN1, Myc-LRRK2-WT, or

Myc-LRRK2-KD alone or in combination. Cell lysates were separated on a phos-tag gel, followed by western blotting with an anti-HA antibody (phos-tag). Expression of proteins in cell lysates was identified using western blotting with anti-Myc or anti-HA antibodies (non-phos-tag). GAPDH served as a loading control. (C) HEK293 cells were transfected for $24 \mathrm{~h}$ with plasmids encoding Myc-tagged LRRK2-WT, or the LRRK2-G2019S or LRRK2-R1441C mutants, and in vitro kinase assay was performed. The intensity of phosphorylated RCAN1 bands was quantified by densitometry using Multi Gauge v3.1 software (right panel in C,D). Individual band intensities were normalized to that for total RCAN1 for each experimental group. Error bars indicate \pm SEM in triplicate experiments $\left({ }^{*} p<0.05 ;{ }^{* *} p<0.01\right)$. (D) HEK293/LL-1Rl cells were mock-transfected or transfected for $24 \mathrm{~h}$ with Myc-LRRK2-WT, and treated with $10 \mathrm{ng} / \mathrm{ml} \mathrm{IL}-1 \beta$ for the indicated times. Cell lysates were immunoprecipitated with an anti-Myc antibody, and in vitro kinase assay was performed. (E) Schematic representation of wild-type RCAN1, its deletion mutants, and a summary of the phosphorylation assay results. (F,G) After HEK293 cells were mock-transfected or transfected for $24 \mathrm{~h}$ with Myc-LRRK2-WT, in vitro kinase assay was performed with GST-fused RCAN1 deletion mutants, as indicated. Asterisks indicate non-specific proteins. (H-J) HEK293 cells were mock-transfected or transfected for $24 \mathrm{~h}$ with Myc-LRRK2-WT, and in vitro kinase assay was performed with recombinant GST-RCAN1-WT and GST-RCAN1-S162A (H), GST-RCAN1-WT, GST-RCAN1-S149A, RCAN1-T151A, RCAN1-T152A, and RCAN1-T154A (I), or GST-RAB5B (J) as a substrate.

in response to treatment with wild-type LRRK2 and two PD-associated mutants. Co-IP analyses showed that the mutants LRRK2-G2019S and LRRK2-R1441C increased formation of RCAN1-Tollip complexes, much more than did wild-type LRRK2 (Figure 4E). These data are consistent with findings regarding LRRK2-mediated RCAN1 phosphorylation. They also suggest that the binding of RCAN1 to Tollip is dependent upon the kinase activity of LRRK2.
To determine whether the interaction between RCAN1 and Tollip is associated with the progression of $\mathrm{PD}$, we tested the effect of neurotoxin, $\mathrm{MPP}^{+}$, which causes symptoms of $\mathrm{PD}$ by destroying dopaminergic neurons in the substantia nigra of the brain, on LRRK2-induced RCAN1-Tollip interaction in neuroblastoma SH-SY5Y cell line. Interestingly, $\mathrm{MPP}^{+}$treatment further increased the binding affinity between RCAN1 and Tollip which is stimulated by LRRK2 (Figure 4F). These results suggest that the formation of RCAN1-Tollip complex which 


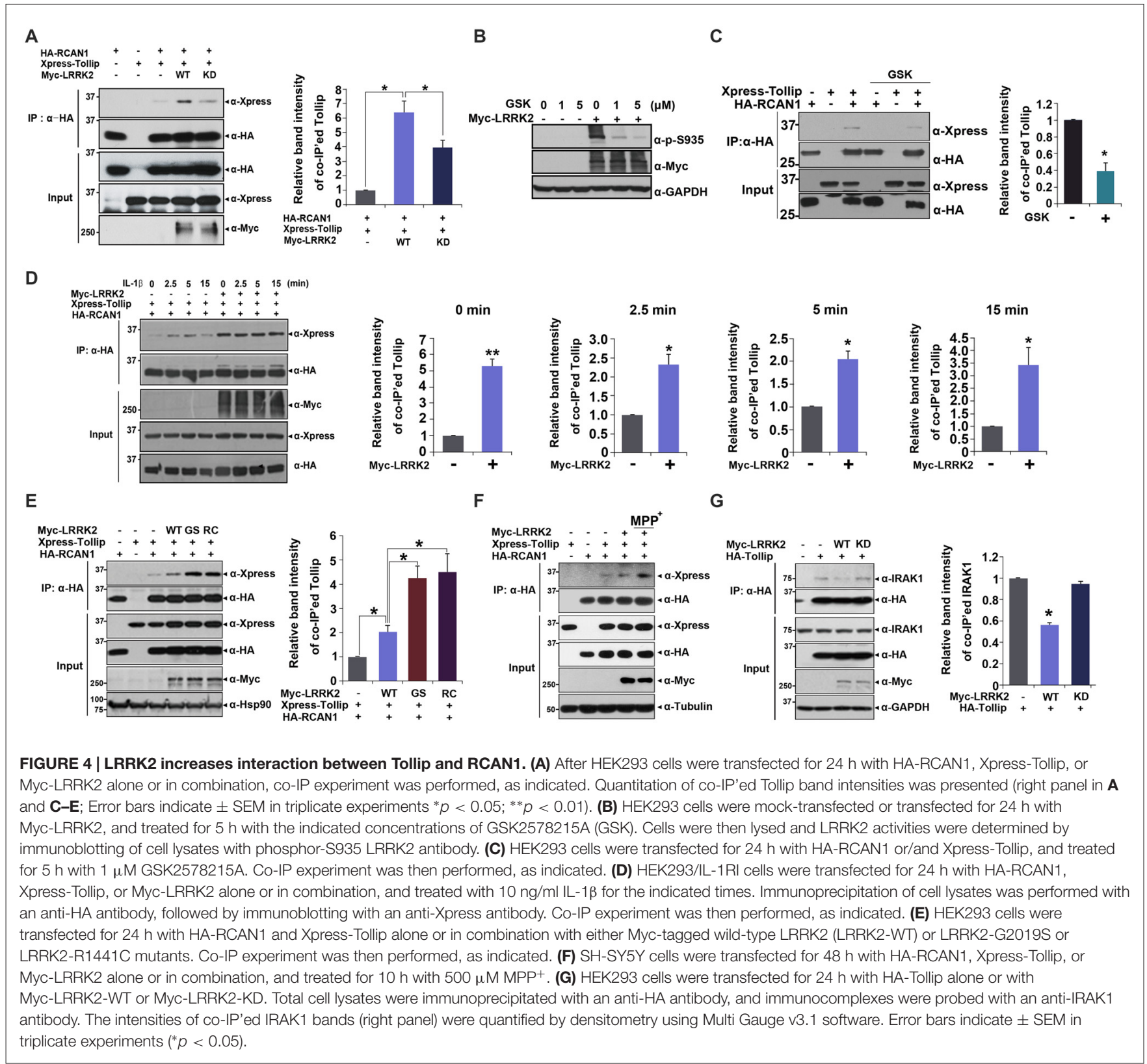

is affected by LRRK2 might play a role in the pathogenesis of PD.

In addition to modulation of IL-1R-binding complex formation, Tollip also binds to IRAK1 and acts as a negative modulator of downstream IRAK1 signaling. Thus, we further examined whether LRRK2 can regulate the formation of Tollip-IRAK1 complexes. HEK293/IL-1RI cells were transfected with HA-Tollip alone or with Myc-tagged LRRK2-WT or the LRRK2-KD mutant; cell lysates were immunoprecipitated using an anti-HA antibody. Immunoblot analyses of anti-HA immunocomplexes using an anti-IRAK1 antibody showed that LRRK2 promoted the dissociation of Tollip-IRAK1 complexes (Figure 4G). These results suggest that LRRK2 positively regulates association between RCAN1 and Tollip, while inhibiting association between Tollip and IRAK1, which consequently potentiates IL-1R-mediated downstream signaling.

\section{LRRK2 Promotes the Assembly of IRAK1-TRAF6}

Stimulation of IL-1R/TLR with IL-1 $\beta$ results in activation of IRAK1 and subsequent recruitment of TRAF6 to IRAK1 complexes (Jiang et al., 2002). The IRAK1TRAF6 complex dissociates from the activated heterodimer receptor. Activated IRAK1 is degraded by the UPS through the Skp1-Cullin1-F-box- $\beta$-TrCP complex, while TRAF6 activates downstream inflammatory signaling (Cui et al., 2012). Previous reports also indicate that K63-linked ubiquitination of 
IRAK1 occurs during stimulation with IL-1, and that it is

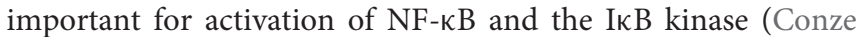
et al., 2008; Windheim et al., 2008).

Based on these observations, we next investigated whether LRRK2 also modulates the assembly of IRAK1TRAF6 complexes. HEK293/IL-1RI cells were mock-transfected or transfected with Myc-tagged LRRK2-WT or Myc-LRRK2$\mathrm{KD}$ mutant for $24 \mathrm{~h}$. Cells were then treated for $6 \mathrm{~h}$ with the proteasome inhibitor MG132 to prevent IRAK1 degradation and for $2.5,5$, or $20 \mathrm{~min}$ with IL-1 $\beta$. Immunoprecipitation of cell lysates was performed using an anti-TRAF6 antibody, followed by immunoblotting with anti-IRAK1 antibodies. LRRK2-WT enhanced the association of IRAK1 and TRAF6 at 2.5 and 5 min after IL- $1 \beta$ treatment; the LRRK2-KD mutant had no significant effect on this interaction (Figure 5A). Consistent with this finding, when cells were treated with MG132, we also observed formation of smeared IRAK1 bands, indicating K48and K63-linked polyubiquitination of IRAK1 in response to IL-1 $\beta$ stimulation (Figure 5A).

We next examined whether LRRK2 deletion inversely affects interaction between endogenous IRAK1 and endogenous TRAF6. After treatment of LRRK2 +/+ and LRRK2 -/- MEFs with IL-1 $\beta$, we evaluated IRAK1-TRAF6 complex formation. Co-IP analyses demonstrated that levels of endogenous IRAK1-TRAF6 complexes were significantly reduced in LRRK2 -/ - MEF after stimulation with IL-1 $\beta$, compared with LRRK2 +/+ MEFs (Figure 5B). These data indicate that LRRK2 facilitates formation of IRAK1-TRAF6 complexes in response to IL- $1 \beta$ stimulation.

To further investigate the specific effects of LRRK2 on RCAN1 function in response to IL- $1 \beta$ stimulation, we utilized RCAN1 $1^{1-145}$ deletion mutant, which is not phosphorylated by LRRK2. Unlike the stimulatory effect of RCAN1-WT, the RCAN1 ${ }^{1-145}$ fragment failed to increase the interaction between IRAK1 and TRAF6 (Figure 5C). These results indicate that LRRK2 stimulates inflammatory signaling through potentiation of RCAN1-mediated positive modulation of IL-1 $\beta$ signaling.

\section{LRRK2 Enhances the Auto-Phosphorylation of TAK1}

Upon stimulation with IL-1 $\beta$, K63-linked polyubiquitination of TRAF6 occurs after IRAK1-TRAF6 complex formation, which subsequently activates TAK1 (Sun et al., 2004). Based on
A
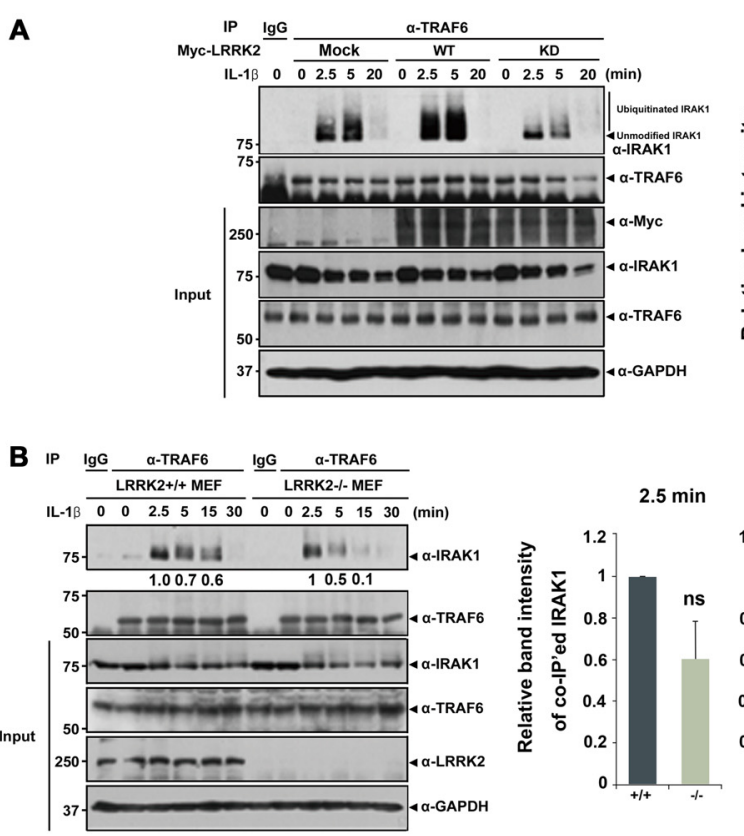
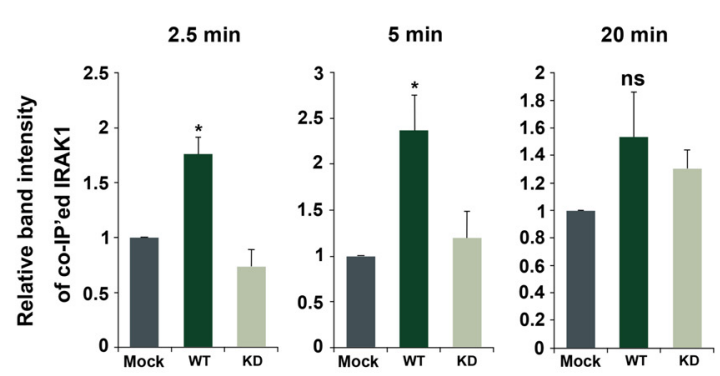

C
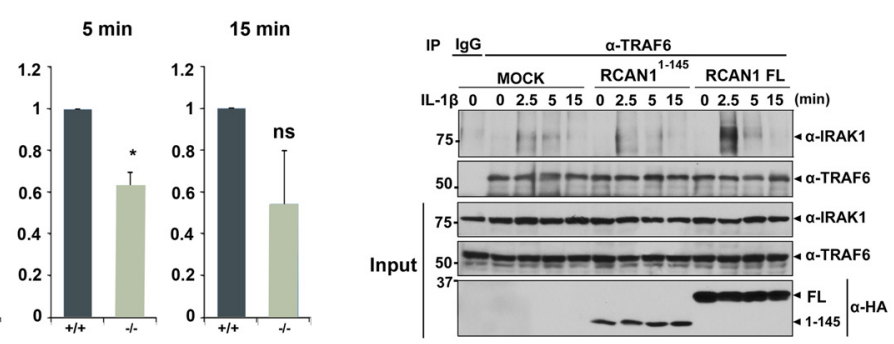

FIGURE 5 | LRRK2 facilitates assembly of the IRAK1-TRAF6 complex. (A) HEK293/L-1RI cells were either mock-transfected or transfected for $24 \mathrm{~h}$ with Myc-tagged LRRK2-WT or the LRRK2-KD mutant and treated with $10 \mathrm{ng} / \mathrm{ml} \mathrm{IL}-1 \beta$ for the indicated times. Cell lysates were immunoprecipitated with an anti-TRAF6 antibody and immunoblotted with an anti-IRAK1 antibody. Expression of Myc-LRRK2-WT, Myc-LRRK2-KD, endogenous TRAF6, and IRAK1 in cell lysates was determined using immunoblotting with anti-Myc, anti-TRAF6, or anti-IRAK1 antibodies (left panel). (B) LRRK2 +/+ or LRRK2 -/- Mouse embryonic fibroblasts (MEFs) were treated with $50 \mathrm{ng} / \mathrm{ml} \mathrm{IL-1 \beta}$ for the indicated times. Cell lysates were immunoprecipitated with an anti-TRAF6 antibody and immunoblotted with an anti-IRAK1 antibody. As a control, cell lysates were immunoprecipitated with pre-immune IgG (lgG), as indicated. Endogenous LRRK2, TRAF6 and IRAK1 levels were determined using immunoblotting with their respective antibodies (left panel). The intensity of co-IP'ed IRAK1 bands was quantified by densitometry using Multi Gauge v3.1 software (right panel in $\mathbf{A}, \mathbf{B} ; n=3$ ). Individual band intensities were normalized to that for the co-IP'ed TRAF6 for each experimental group. Error bars indicate \pm SEM in triplicate experiments $\left({ }^{*} p<0.05\right)$. (C) HEK293/L-1RI cells were either mock-transfected or transfected for $24 \mathrm{~h}$ with HA-tagged full-length RCAN1 (RCAN1-FL) or RCAN1 ${ }^{1-145}$ mutant, and treated with $10 \mathrm{ng} / \mathrm{ml} \mathrm{IL-1 \beta}$ for the indicated times. Cell lysates were immunoprecipitated with anti-TRAF6 antibody and immunoblotted with anti-IRAK1 antibody. Expression of HA-RCAN1-FL, HA-RCAN1 ${ }^{1-145}$, endogenous TRAF6, or IRAK1 in cell lysates was determined using immunoblotting with anti-HA, anti-TRAF6, or anti-IRAK1 antibodies. 
reports that TAK1 auto-phosphorylation at Thr187 is closely related to its kinase activity (Kajino et al., 2006; Yu et al., 2008), we next investigated the effects of LRRK2 on basal and IL- $1 \beta$-induced TAK1 activity. After transfection with XpressTollip, HA-RCAN1, or Myc-LRRK2 alone or in combination, HEK293/IL-1RI cells were left untreated or stimulated with IL-1 $\beta$. Immunoblot analyses of cell lysates with anti-phosphoTAK1 antibodies confirmed phosphorylation of TAK1 at T187 in response to IL-1 $\beta$ stimulation; there was no significant activation of TAK1 in the absence of IL- $1 \beta$. Treatment with IL- $1 \beta$ led to a significant induction of TAK1 phosphorylation, which reached a maximum level after $15 \mathrm{~min}$. When cells were co-transfected with Tollip and RCAN1, TAK1 activity was more elevated compared with when cells were transfected with Tollip alone. Furthermore, LRRK2 over-expression increased TAK1 activity under resting conditions and after IL- $1 \beta$ stimulation (Figure 6A). Interestingly, LRRK2 overexpression caused much stronger TAK1 phosphorylation, reaching its maximum at $5 \mathrm{~min}$ after IL-1 $\beta$ stimulation (Figure 6B). However, this effect was greatly diminished by RCAN1 knockdown (Figure 6C). These data suggest that RCAN1 could be a key regulator of LRRK2-induced IL- $1 \beta$ signaling.

We further examined the effect of LRRK2 knockdown on TAK1 activity using LRRK2 -/- and LRRK2 +/+ MEFs. Immunoblot analyses of cell lysates with a phospho-TAK1 antibody showed that TAK1 activity was significantly decreased in LRRK2 -/ - MEF compared with LRRK2 +/+ MEF (Figure 6D). When we investigated the effects of two PD-associated LRRK2 mutants (LRRK2-G2019S and LRRK2-R1441C) on TAK1 activity, both mutants enhanced
A

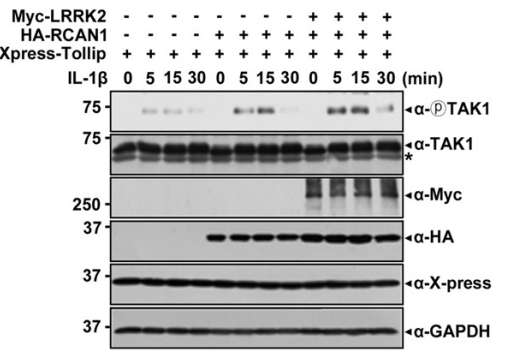

C

Myc-LRRK2 $\frac{\text { si-RCAN1 }}{+++++\cdots \cdots} \frac{\text { nc si-RNA }}{+++++}$

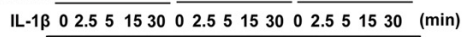
75 75 - $20-$ TAK1

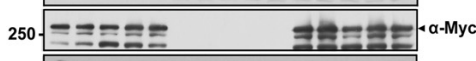
25 - $-\alpha-$ RCAN1

B
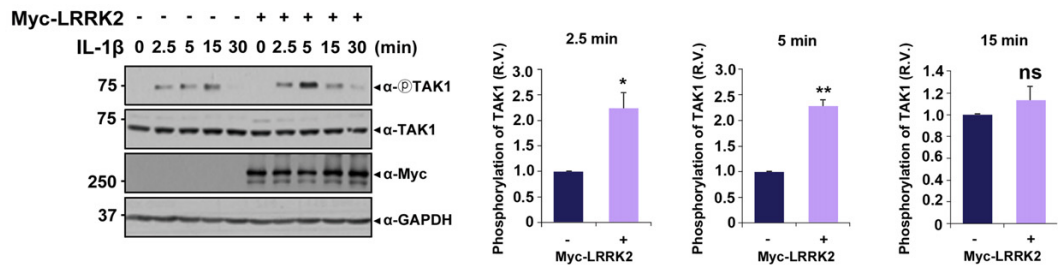

D
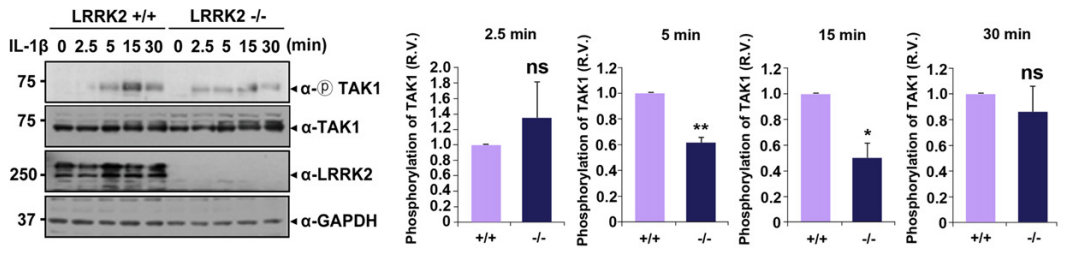

E
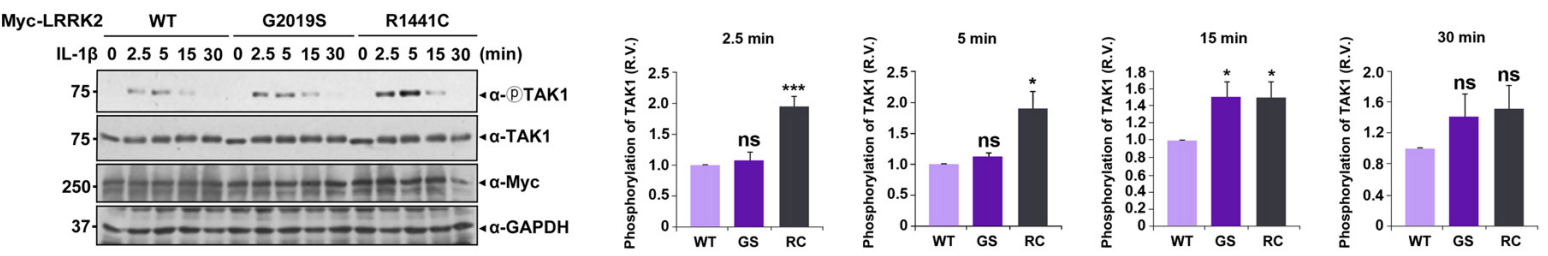

FIGURE 6 | LRRK2 potentiates TAK1 auto-phosphorylation in response to IL-1 $\beta$ stimulation. (A) HEK293/L-1RI cells were transfected for $24 \mathrm{~h}$ with Xpress-Tollip, HA-RCAN1, or Myc-LRRK2 alone or in combination and treated with $10 \mathrm{ng} / \mathrm{ml} \mathrm{IL}-1 \beta$ for the indicated times. Cell lysates were immunoblotted with anti-phospho-TAK1 (Thr187), anti-TAK1, or anti-LRRK2 antibodies. GAPDH served as a loading control. Asterisks indicate non-specific proteins. (B) HEK293/L-1RI cells were mock-transfected or transfected for $24 \mathrm{~h}$ with Myc-LRRK2 and treated with $10 \mathrm{ng} / \mathrm{ml} \mathrm{IL-1 \beta}$ for the indicated times. Cell lysates were immunoblotted with anti-phospho-TAK1 (Thr187), anti-TAK1, or anti-LRRK2 antibodies. GAPDH served as a loading control (left panel). Quantitation of the images was presented (right panel). Error bars indicate \pm SEM in triplicate experiments $\left({ }^{*} p<0.05 ;{ }^{* *} p<0.01\right)$. (C) Where specified, HEK293/L-1RI cells were mock-transfected or transfected for $24 \mathrm{~h}$ with nonspecific control si-RNA (nc-si-RNA; $200 \mathrm{nM}$ ), RCAN1-siRNA (si-RCAN1; $200 \mathrm{nM}$ ), or Myc-LRRK2-WT alone or in combination. Cells were then treated with $10 \mathrm{ng} / \mathrm{ml} \mathrm{IL-1 \beta}$ for the indicated times. RCAN1 knockdown was verified using western blotting with an anti-RCAN1 antibody. Cell lysates were immunoblotted with anti-phospho-TAK1 (Thr187), anti-TAK1, or anti-Myc antibodies. GAPDH served as a loading control. (D) LRRK2 +/+ or LRRK2 -/- MEFs were treated with $50 \mathrm{ng} / \mathrm{ml} \mathrm{IL-1 \beta}$ for the indicated times. Cell lysates were immunoblotted with anti-phospho-TAK1 (Thr187), anti-TAK1, or anti-LRRK2 antibodies. GAPDH served as a loading control (left panel). The intensity of phospho-TAK1 bands was quantified by densitometry using Multi Gauge v3.1 software (right panel; $n=3$ in $\mathbf{D}$ and $n=4$ in $\mathbf{E}$ ). Individual band intensities were normalized to that for total TAK1 for each experimental group. Error bars indicate \pm SEM in triplicate experiments $\left({ }^{*} p<0.05,{ }^{* *} p<0.01\right.$ in $\mathbf{D}$; and ${ }^{*} p<0.05,{ }^{* * *} p<0.001$ in E). (E) HEK293/L-1RI cells were transfected for $24 \mathrm{~h}$ with plasmids encoding Myc-tagged LRRK2-WT or the LRRK2-G2019S and LRRK2-R1441C mutants, and then treated with $10 \mathrm{ng} / \mathrm{ml} \mathrm{IL-1 \beta}$ for the indicated times. Cell lysates were immunoblotted with anti-phospho-TAK1 (Thr187), anti-TAK1, or anti-Myc antibodies. GAPDH served as a loading control. 
TAK1 activity (Figure 6E), consistent with other results reported herein. Collectively, these data suggest that LRRK2 potentiates TAK1 activity in response to treatment with IL-1 $\beta$, which consequently stimulates IL- $1 \beta$ mediated downstream signaling.
LRRK2 Enhances Production of IL-8 and Activation of NF- $\kappa$ B in Response to IL-1 $\beta$

Stimulation with IL- $1 \beta$ elicits transcriptional activation of NF- $\kappa B$ in target cells (Yamazaki et al., 2009; Fan et al., 2010). To

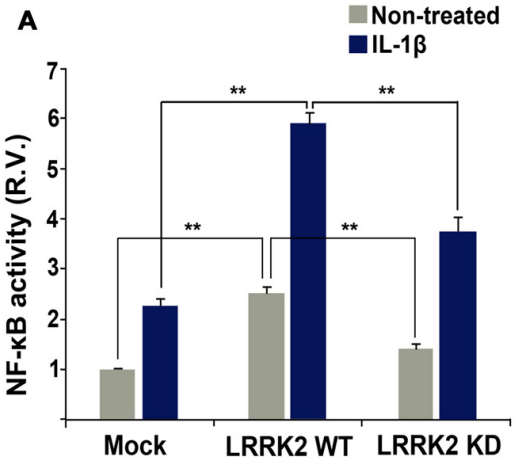

D

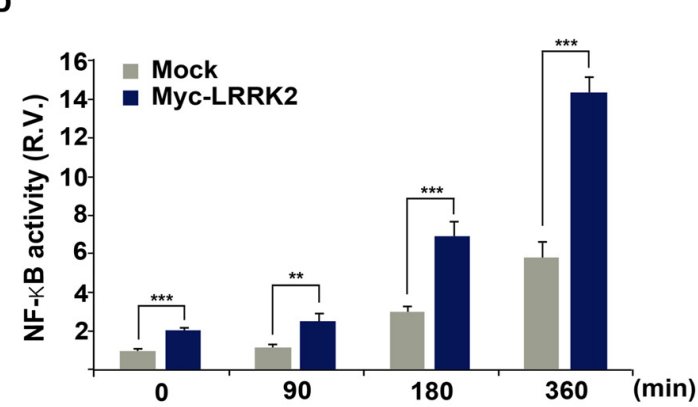

B

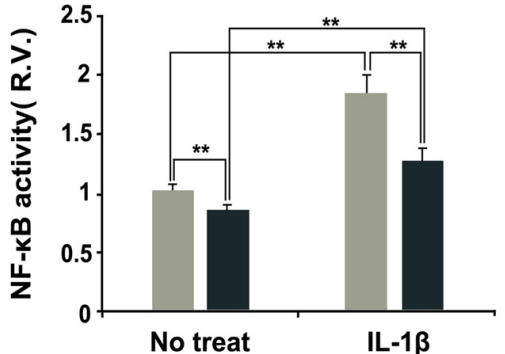

C

C LRRK2 -/- + Myc-LRRK2

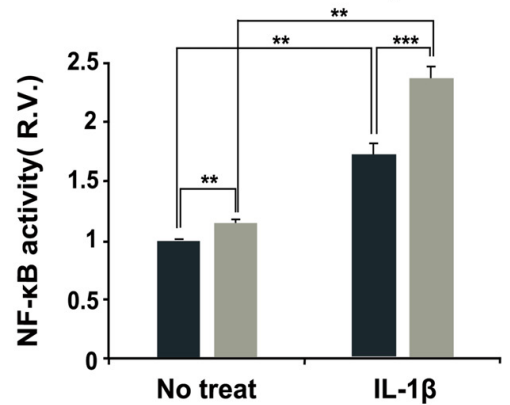

$\mathbf{F}$

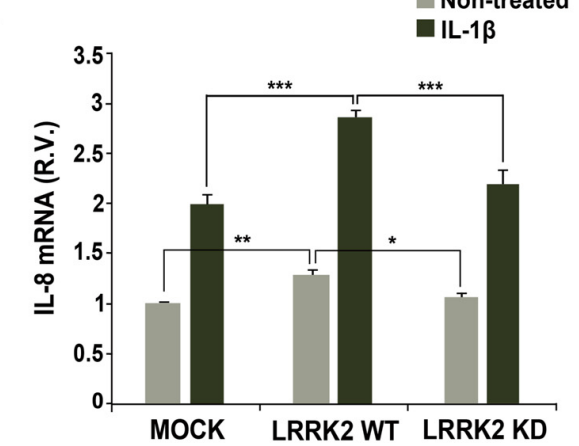

$\mathbf{G}$

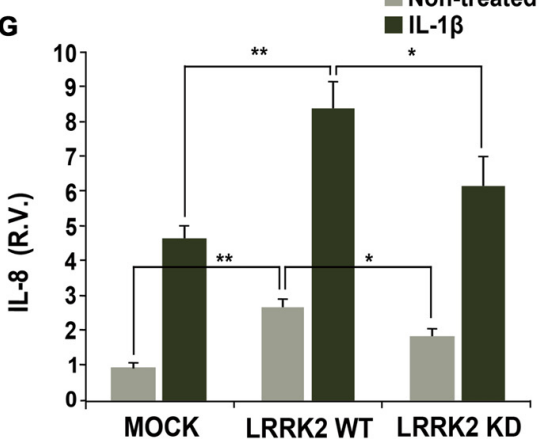

E

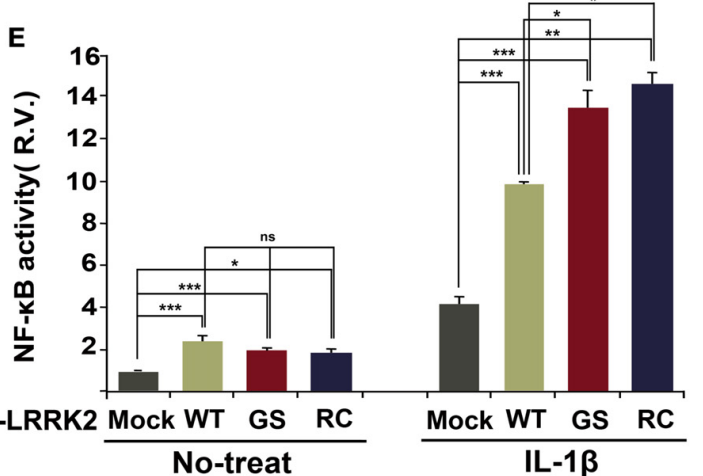

H

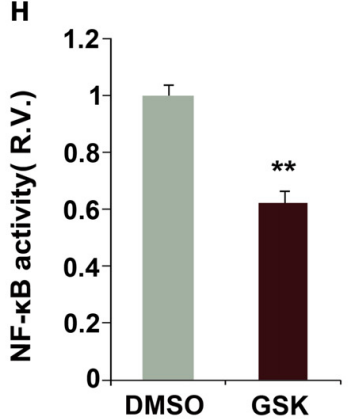

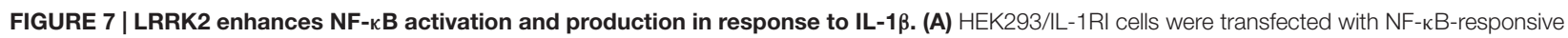
luciferase reporter and a control Renilla luciferase reporter alone or in combination with Myc-LRRK2-WT or Myc-LRRK2-D1994A (LRRK2-KD). Cells were then treated for $6 \mathrm{~h}$ with $10 \mathrm{ng} / \mathrm{ml} \mathrm{IL-1 \beta}$. Relative luciferase activity was measured and normalized to Renilla activity. Error bars indicate \pm SEM in triplicate experiments $\left(\mathbf{A}, \mathbf{B} ;{ }^{* *} p<0.01\right)$. (B,C) Where specified, LRRK2 +/+ and LRRK2 -/- MEFs were transfected with NF-kB-responsive luciferase reporter and a control Renilla luciferase reporter (B). Or else, LRRK2 -/- MEF cells were transfected with NF-kB-responsive luciferase reporter and a control Renilla luciferase reporter alone or in combination with Myc-LRRK2-WT (C). Cells were then treated for $6 \mathrm{~h}$ with $50 \mathrm{ng} / \mathrm{ml} \mathrm{IL}-1 \beta$. The relative luciferase activity was measured and normalized to Renilla activity. (D,E) HEK293/IL-1RI cells were transfected with NF-кB-responsive luciferase reporter and a control Renilla luciferase reporter in combination with empty vector or Myc-LRRK2-WT, or its LRRK2-G2019S or LRRK2-R1441C mutants. Cells were then treated for the indicated times (D) or $6 \mathrm{~h}$ (E) with $10 \mathrm{ng} / \mathrm{ml}$ IL-1 $\beta$. Their relative luciferase activities were measured and normalized to Renilla activity $\left(\mathbf{D}, \mathbf{E} ;{ }^{*} p<0.05 ;{ }^{* *} p<0.01 ;{ }^{* * *} p<0.001\right)$. (F,G) HEK293 cells were either mock-transfected or transfected with Myc-LRRK2-WT or Myc-LRRK2-KD. Cells were then left untreated or stimulated for 8 (F) or 24 h (G) with 25 ng/ml IL-1 $\beta$. Real-time PCR analysis of IL-8 mRNA was performed using IL-8 primers (F; $\left.{ }^{*} p<0.05 ;{ }^{* *} p<0.01 ; * * *<0.001\right)$. Otherwise, the amount of secreted IL-8 protein was measured by enzyme-linked immunosorbent assay (ELISA) kit (G; $\left.{ }^{*} p<0.05 ;{ }^{* *} p<0.01\right)$. Results are representative of six and five independent experiments. (H) RAW264.7 cells were transfected with NF-кB-responsive luciferase reporter and a control Renilla luciferase reporter, and treated for additional $5 \mathrm{~h}$ with $5 \mu \mathrm{M}$ GSK2578215A (GSK). The relative luciferase activity was measured and normalized to Renilla activity. Error bars indicate \pm SEM in triplicate experiments (** $p<0.01)$. 
investigate potential effects of LRRK2 on NF- $\mathrm{B}$ activity, a luciferase NF- $\kappa \mathrm{B}$ reporter gene assay was performed in the absence or presence of LRRK2. Over-expression of wild-type LRRK2 dramatically enhanced NF- $\kappa \mathrm{B}$ activity in response to IL-1 $\beta$ treatment, but this effect was not observed with LRRK2-KD (Figure 7A). To further confirm the stimulatory effect of LRRK2 on NF- $\kappa$ B activity, we compared the activity of $\mathrm{NF}-\kappa \mathrm{B}$ in LRRK2 -/- and LRRK2 +/+ MEFs. NF- $\kappa \mathrm{B}$ activity in LRRK2 -/ - MEFs was reduced compared with $L R R K 2+/+$ MEFs in response to IL- $1 \beta$ treatment (Figure 7B). In addition, the reduced NF- $\kappa \mathrm{B}$ activity was rescued back to the control level by reintroduction of LRRK2-WT into LRRK2 - / - MEFs (Figure 7C), confirming a positive regulatory effect of LRRK2 on NF- $\kappa \mathrm{B}$ activity. We also examined the time-dependence of NF- $\kappa$ B activity with IL-1 $\beta$ treatment. HEK293/IL-1RI cells were mock-transfected or transfected with LRRK2-WT. NF- $\mathrm{B}$ reporter dual luciferase assays showed that NF- $\kappa \mathrm{B}$ activity more rapidly increased in the presence of LRRK2 (Figure 7D). We additionally assessed the effects of $\mathrm{PD}$-associated pathogenic LRRK2 mutants (LRRK2-G2019S and LRRK2-R1441C) on NF- $\kappa \mathrm{B}$ activity. Consistent with our previous findings that LRRK2 stimulates IL-1 $\beta$-mediated downstream signaling, the two PD-associated mutants, having enhanced kinase activities, stimulated NF- $\kappa \mathrm{B}$ activity more than did their wild-type counterpart (Figure 7E).

Lastly, we investigated whether the positive effect of LRRK2 on IL-1R signaling events leads to the production and subsequent secretion of inflammatory cytokines, such as IL-8, in HEK293/IL-1RI cells in response to stimulation with IL-1 $\beta$. Consistent with the stimulatory effect of LRRK2 on the upstream components of IL-1R signaling, the presence of wild-type LRRK2 increased levels of IL-8 mRNA, as determined by real-time PCR (Figure 7F). Additionally, wild-type LRRK2 stimulated secretion of IL-8, as determined by ELISA (Figure 7G). Interestingly, when compared with mock-transfected controls, cells transfected with LRRK2-WT displayed enhanced NF- $\kappa \mathrm{B}$ activity in the absence of IL-1 $\beta$ treatment, which greatly increased with IL-1 $\beta$ treatment (Figures 7A,E). However, this effect was not observed in cells transfected with LRRK2-KD (Figure 7A). Similarly, the synthesis of IL-8 mRNA and protein greatly increased in cells transfected with LRRK2-WT alone, but not with LRRK-KD (Figures 7F,G). We next examined whether LRRK2 is necessary for enhanced NF- $\kappa \mathrm{B}$ activity in immune cells as well as in HEK293/IL-1RI cells and MEFs. Pretreatment of RAW264.7 cells with LRRK2 inhibitor caused a decrease in NF- $\kappa \mathrm{B}$ activity (Figure 7H). Collectively, these results suggest that LRRK2 acts as a positive regulator of IL- $1 \beta$-mediated signaling, leading to up-regulation of inflammatory cytokines in response to stimulation with IL- $1 \beta$.

\section{DISCUSSION}

The present study demonstrated that PD-associated LRRK2 positively modulates IL- $1 \beta$-mediated inflammatory signaling through selective phosphorylation of RCAN1. Accordingly, excessive brain inflammation is increasingly recognized as a major factor participating in brain diseases, including Alzheimer's disease (AD) and PD. Numerous reports revealed that inflammatory process by immune cell elicits neuronal and non-neuronal cell death in brain (Broughton et al., 2013). Not only that, some molecules closely associated with inflammatory processes, such as serum homocysteine, uric acid, plasma cystatin C, and high-density lipoprotein are commonly regarded as the biomarkers of multiple system atrophy, $\mathrm{AD}$, and vascular dementia (Chen et al., 2015; Wang et al., 2017). Furthermore, inflammation seems to be important for the pathogenesis of $\mathrm{PD}$. For example, neurotoxin $\mathrm{MPP}^{+}$treatment, which causes the dopaminergic neuron loss and PD-like symptoms, promotes the production and release of various cytokines through TLR4 signaling in BV2 cells (Zhou et al., 2016). In addition, many reports suggest that specific molecules and their receptors are closely related to and regulate the progression of PD. For example, Nur77, an important regulator of neuroinflammation and dopaminergic neurodegeneration, seems to play a role in 6-hydroxydopamine (OHDA)-induced model of PD (Gao et al., 2016). Treatment of 6-OHDA changed intracellular localization of Nur77 from nucleus to cytosol, triggering its transcriptional activation and subsequent cell death via aggravating mitochondrial impairment and endoplasmic reticulum stress (Gao et al., 2016). Or else, memantine, an NMDA receptor antagonist and commonly used AD medication, suppresses the expression of Nur77 and Nurr1 through anti-inflammation and anti-mitochondrial impairment (Wei et al., 2016). The nuclear translocation of Nur77 was also reduced by memantine (Gao et al., 2016; Wei et al., 2016). Anti-inflammatory agent, simvastatin, also affects dopaminergic neuronal degeneration. For example, simvastatin suppressed 6-OHDA-induced neurotoxicity and increased the levels of IL-6, TNF- $\alpha$ and $\operatorname{COX}_{2}$ (Xu et al., 2013). Moreover, the functional and pathological alteration of adrenergic receptors, acetylcholine receptors, and dopamine receptors contribute cognitive deficits in $\mathrm{PD}$ patients, underlying the involvement of variety signaling pathways ( $\mathrm{Xu}$ et al., 2012). All these reports including the current study further validate the hypothesis that inflammation is an important factor for PD.

Several studies have recently reported a mechanistic link between LRRK2 and innate immunity and propose a functional role for LRRK2 in the positive regulation of NF- $\kappa$ B-dependent gene transcription (Kim et al., 2007; Gardet et al., 2010). Despite intensive investigation, the underlying mechanism of LRRK2-mediated inflammatory response and the signaling pathways involved have not yet been clearly elucidated. Two putative upstream regulators of LRRK2 have been identified: ERK5 and IKK. Activation of LRRK2 can be induced through ERK5 signaling by treatment with interferon- $\gamma$ (Kuss et al., 2014). Additionally, exposure to LPS increases levels of phosphorylated LRRK2, whereas treatment with IKK inhibitors inhibits LRRK2 phosphorylation (Dzamko et al., 2012). While these two proteins appear to act upstream of LRRK2, there is not much known about downstream substrates 
of LRRK2 during IL-1R/TLR signaling. Here, we present several lines of evidence demonstrating that RCAN1 positively regulates in IL- $1 \beta$ signaling pathway, which is potentiated by LRRK2-mediated phosphorylation of RCAN1. We show that LRRK2 physically interacts with RCAN1 and that the N-terminal 30-95 amino acid region of RCAN1 is critical for their interaction. LRRK2 also directly phosphorylates RCAN1, which is greatly increased with stimulation by IL-1 $\beta$.

Like others proteins, RCAN1 is modulated by various post-translational modifications. Among them, phosphorylation is the most prominent regulatory mechanism. A number of protein kinases regulate the functional activity and/or stability of RCAN1. For example, protein kinase $\mathrm{A}$ and NF-кBinducing kinase phosphorylate RCAN1 and increase its halflife, which consequently enhances the inhibitory function of RCAN1 in NFAT signaling (Lee et al., 2008; Kim et al., 2012). In addition, Dyrk1A also promotes the inhibitory effect of RCAN1 on calcineurin through its kinase activity (Jung et al., 2011). In contrast, MEK5-BMK1 signaling and TAK1 suppress RCAN1 function, increasing NFAT activity (Abbasi et al., 2006; Liu et al., 2009). Furthermore, the yeast protein Mck1, a member of the GSK-3 family of protein kinases, phosphorylates Rcn1, enhancing downstream calcineurin signaling (Hilioti et al., 2004). Here, we identified LRRK2 as a novel kinase acting on RCAN1. Unlike other kinases, LRRK2 has no effect on RCAN1 protein stability. While we could not determine the exact sites of RCAN1 targeted by LRRK2, it may modify other sites distinct from those of other kinases. Further experimentation is required to investigate this hypothesis.

The protein binding partners of RCAN1 are classified into two groups, depending on their involvement in the calcineurin signaling pathway. For example, RCAN1 binds to the cAMP response element-binding protein (CREB) and positively regulates the CREB pathway via modulation of calcineurin activity (Kim and Seo, 2011). Under oxidative stress conditions, RCAN1 plays a protective role through regulation of the CREB pathway (Kim et al., 2013). Furthermore, RCAN1 negatively regulates vesicle recycling and endocytosis via inhibition of calcineurin activity (Zanin et al., 2013). As example of a binding partner not associated with calcineurin activity is the interaction between RCAN1 and phospho-FMRP, which induces spine morphogenesis and local protein synthesis in dendritic spines (Wang et al., 2012). We previously showed that the binding of RCAN1 to Tollip, an inhibitor of IL-1 $\beta$ signaling, potentiates IL-1 $\beta$-mediated transduction (Lee et al., 2009), which also occurs through a calcineurin-independent pathway. The present study reveals that LRRK2 falls under the second group of RCAN1-binding proteins. Moreover, LRRK2 does not affect RCAN1 binding to calcineurin, nor downstream NFAT activity (Han and Chung, unpublished observation). These results suggest that LRRK2 modulates RCAN1 independently from downstream calcineurin-signaling pathway.

In the present work, we demonstrate that LRRK2 increase binding of RCAN1 to Tollip. LRRK2 stimulated the downstream formation of a signalosome complex involving IRAK1 and TRAF6, and facilitated TAK1 auto-phosphorylation in response to stimulation by IL- $1 \beta$. Consistent with other present findings, LRRK2 dramatically increased NF- $\mathrm{KB}$ activity in response to IL-1 $\beta$ treatment. Furthermore, LRRK2 potentiated the production of IL-8 and its extracellular release via NF- $\mathrm{\kappa B}$ activation in response to IL- $1 \beta$ treatment. According to the previous report, myeloid cell recruitment was reduced in LRRK2-knockout rat by LPS treatment, leading to the suppression of dopaminergic neurodegeneration (Daher et al., 2014). The present work also suggests that chemokine IL-8 release could play a role in LRRK2-mediated toxicity by recruiting the myeloid cells to midbrain region. In contrast to our present findings, previous studies reported that the kinase-dead LRRK2 mutant and its wild-type counterpart both promote NF-кB activity (Kim et al., 2007; Gardet et al., 2010). This discrepancy may be due to the different stimuli and cell types used. Therefore, other immune cell types should be tested. However, our findings were corroborated by data demonstrating that two PD-associated LRRK2 mutants, both possessing enhanced kinase activity, correspondingly increased phosphorylation of RCAN1 compared with wild-type LRRK2. These results also provide additional insight into the role of these pathogenic LRRK2 mutations during putative RCAN1mediated IL-1-induced inflammation in PD. Our results may serve as a basis for future work in RCAN1 knockout/transgenic models and PD-associated models over-expressing these LRRK2 mutants.

Inflammation and the inflammatory response are important contributing factors to the pathogenesis of various neurodegenerative diseases, including PD. Collectively, the present work suggests that alteration of LRRK2 function might play a significant role in the development of these conditions.

\section{AUTHOR CONTRIBUTIONS}

$\mathrm{KAH}$ and KCC formulated the hypothesis, initiated and organized the study and wrote the manuscript. KAH, JWU, HK, WS and KCC analyzed data. KAH, JYS, LY and SAC performed experiments.

\section{FUNDING}

This work was supported by grants from the National Research Foundation of Korea (NRF) funded by the Ministry of Science, ICT and Future Planning (2014M3C7A1064545 and 2015R1A2A2A01003080 to KCC), South Korea. This work was also supported in part by the Yonsei University Future-leading Research Initiative of 2015 (2015-22-0055 to KCC).

\section{ACKNOWLEDGMENTS}

The authors thank S. de la Luna, D.R. Alessi, and G. Takaesu for providing plasmids and cell lines. 


\section{REFERENCES}

Abbasi, S., Lee, J. D., Su, B., Chen, X., Alcon, J. L., Yang, J., et al. (2006). Protein kinase-mediated regulation of calcineurin through the phosphorylation of modulatory calcineurin-interacting protein 1. J. Biol. Chem. 281, 7717-7726. doi: 10.1074/jbc.M510775200

Broughton, B. R., Lim, R., Arumugam, T. V., Drummond, G. R., Wallace, E. M., and Sobey, C. G. (2013). Post-stroke inflammation and the potential efficacy of novel stem cell therapies: Focus on amnion epithelial cells. Front. Cell. Neurosci. 6:66. doi: 10.3389/fncel.2012. 00066

Cao, Z., Henzel, W. J., and Gao, X. (1996). IRAK: a kinase associated with the interleukin-1 receptor. Science 271, 1128-1131. doi: 10.1126/science.271.52 52.1128

Chen, D., Wei, X., Zou, J., Wang, R., Liu, X., Xu, X., et al. (2015). Contradirectional expression of serum homocysteine and uric acid as important biomarkers of multiple system atrophy severity: a cross-sectional study. Front. Cell. Neurosci. 6:247. doi: 10.3389/fncel.2015.00247

Conze, D. B., Wu, C. J., Thomas, J. A., Landstrom, A., and Ashwell, J. D. (2008). Lys63-linked polyubiquitination of IRAK-1 is required for interleukin-1 receptor- and toll-like receptor-mediated NF-кB activation. Mol. Cell. Biol. 28, 3538-3547. doi: 10.1128/MCB.02098-07

Cui, W., Xiao, N., Xiao, H., Zhou, H., Yu, M., Gu, J., et al. (2012). $\beta$-TrCPmediated IRAK1 degradation releases TAK1-TRAF6 from the membrane to the cytosol for TAK1-dependent NF-кB activation. Mol. Cell. Biol. 32, 3990-4000. doi: 10.1128/MCB.00722-12

Daher, J. P., Volpicelli-Daley, L. A., Blackburn, J. P., Moehle, M. S., and West, A. B. (2014). Abrogation of $\alpha$-synuclein-mediated dopaminergic neurodegeneration in LRRK2-deficient rats. Proc. Natl. Acad. Sci. U S A 111, 9289-9294. doi: 10.1073/pnas.1403215111

Deng, X., Dzamko, N., Prescott, A., Davies, P., Liu, Q., Yang, Q., et al. (2011). Characterization of a selective inhibitor of the Parkinson's disease kinase LRRK2. Nat. Chem. Biol. 7, 203-205. doi: 10.1038/nchembio.538

Dzamko, N., Inesta-Vaquera, F., Zhang, J., Xie, C., Cai, H., Arthur, S., et al. (2012). The IkappaB kinase family phosphorylates the Parkinson's disease kinase LRRK2 at Ser935 and Ser910 during Toll-like receptor signaling. PLoS One 7:e39132. doi: 10.1371/journal.pone.0039132

Fan, Y., Yu, Y., Shi, Y., Sun, W., Xie, M., Ge, N., et al. (2010). Lysine 63-linked polyubiquitination of TAK1 at lysine 158 is required for tumor necrosis factor $\alpha$ - and interleukin-1 $\beta$-induced IKK/NF-кB and JNK/AP-1 activation. J. Biol. Chem. 285, 5347-5360. doi: 10.1074/jbc.M109.076976

Fuentes, J. J., Genescà, L., Kingsbury, T. J., Cunningham, K. W., Pérez-Riba, M., Estivil, X., et al. (2000). DSCR1, overexpressed in Down syndrome, is an inhibitor of calcineurin-mediated signaling pathways. Hum. Mol. Genet. 9, 1681-1690. doi: 10.1093/hmg/9.11.1681

Gao, H., Chen, Z., Fu, Y., Yang, X., Weng, R., Wang, R., et al. (2016). Nur77 exacerbates PC12 cellular injury in vitro by aggravating mitochondrial impairment and endoplasmic reticulum stress. Sci. Rep. 6:34403. doi: $10.1038 /$ srep34403

Gardet, A., Benita, Y., Li, C., Sands, B. E., Ballester, I., Stevens, C., et al. (2010). LRRK2 is involved in the IFN-gamma response and host response to pathogens. J. Immunol. 185, 5577-5585. doi: 10.4049/jimmunol.1000548

Gillardon, F., Schmid, R., and Draheim, H. (2012). Parkinson's disease-linked leucine-rich repeat kinase $2(\mathrm{R} 1441 \mathrm{G})$ mutation increases proinflammatory cytokine release from activated primary microglial cells and resultant neurotoxicity. Neuroscience 208, 41-48. doi: 10.1016/j.neuroscience.2012. 02.001

Heo, H. Y., Park, J. M., Kim, C. H., Han, B. S., Kim, K. S., and Seol, W. (2010). LRRK2 enhances oxidative stress-induced neurotoxicity via its kinase activity. Exp. Cell Res. 316, 649-656. doi: 10.1016/j.yexcr.2009. 09.014

Hilioti, Z., Gallagher, D. A., Low-Nam, S. T., Ramaswamy, P., Gajer, P., Kingsbury, T. J., et al. (2004). GSK-3 kinases enhance calcineurin signaling by phosphorylation of RCNs. Genes. Dev. 18, 35-47. doi: 10.1101/gad. 1159204

Jiang, Z., Ninomiya-Tsuji, J., Qian, Y., Matsumoto, K., and Li, X. (2002). Interleukin-1 (IL-1) receptor-associated kinase-dependent IL-1-induced signaling complexes phosphorylate TAK1 and TAB2 at the plasma membrane and activate TAK1 in the cytosol. Mol. Cell. Biol. 22, 7158-7167. doi: $10.1128 / \mathrm{mcb} .22 .20 .7158-7167.2002$

Jung, M. S., Park, J. H., Ryu, Y. S., Choi, S. H., Yoon, S. H., Kwen, M. Y., et al. (2011). Regulation of RCAN1 protein activity by Dyrk1A protein-mediated phosphorylation. J. Biol. Chem. 286, 40401-40412. doi: 10.1074/jbc.M111. 253971

Kajino, T., Ren, H., Iemura, S., Natsume, T., Stefansson, B., Brautigan, D. L., et al. (2006). Protein phosphatase 6 down-regulates TAK1 kinase activation in the IL-1 signaling pathway. J. Biol. Chem. 281, 39891-39896. doi: 10.1074/jbc. M608155200

Kim, S. S., Jang, S. A., and Seo, S. R. (2013). CREB-mediated Bcl-2 expression contributes to RCAN1 protection from hydrogen peroxide-induced neuronal death. J. Cell. Biochem. 114, 1115-1123. doi: 10.1002/jcb.24452

Kim, S. S., Oh, Y., Chung, K. C., and Seo, S. R. (2012). Protein kinase A phosphorylates down syndrome critical region 1 (RCAN1). Biochem. Biophys. Res. Commun. 418, 657-661. doi: 10.1016/j.bbrc.2012.01.071

Kim, S. S., and Seo, S. R. (2011). The regulator of calcineurin 1 (RCAN1/DSCR1) activates the cAMP response element-binding protein (CREB) pathway. J. Biol. Chem. 286, 37841-37848. doi: 10.1074/jbc.M111.232165

Kim, B., Yang, M. S., Choi, D., Kim, J. H., Kim, H. S., Seol, W., et al. (2007). Impaired inflammatory responses in murine Lrrk2-knockdown brain microglia. PLoS One 7:e34693. doi: 10.1371/journal.pone.0034693

Kuss, M., Adamopoulou, E., and Kahle, P. J. (2014). Interferon- $\gamma$ induces leucine-rich repeat kinase LRRK2 via extracellular signal-regulated kinase ERK5 in macrophages. J. Neurochem. 129, 980-987. doi: 10.1111/jnc.12668

Lawrence, T. (2009). The nuclear factor NF-кB pathway in inflammation. Cold Spring Harb. Perspect. Biol. 1:a001651. doi: 10.1101/cshperspect. a001651

Lee, J. W., Kang, H. S., Lee, J. Y., Lee, E. J., Rhim, H., Yoon, J. H., et al. (2012). The transcription factor STAT2 enhances proteasomal degradation of RCAN1 through the ubiquitin E3 ligase FBW7. Biochem. Biophys. Res. Commun. 420, 404-410. doi: 10.1016/j.bbrc.2012.03.007

Lee, J. Y., Lee, H. J., Lee, E. J., Jang, S. H., Kim, H., Yoon, J. H., et al. (2009) Down syndrome candidate region-1 protein interacts with Tollip and positively modulates interleukin-1 receptor-mediated signaling. Biochim. Biophys. Acta 1790, 1673-1680. doi: 10.1016/j.bbagen.2009.08.005

Lee, E. J., Seo, S. R., Um, J. W., Park, J., Oh, Y., and Chung, K. C. (2008) NF-кB-inducing kinase phosphorylates and blocks the degradation of down syndrome candidate region 1. J. Biol. Chem. 283, 3392-3400. doi: 10.1074/jbc. M706707200

Li, J. Q., Tan, L., and Yu, J. T. (2014). The role of the LRRK2 gene in Parkinsonism. Mol. Neurodegener. 9:47. doi: 10.1186/1750-1326-9-47

Liu, Q., Busby, J. C., and Molkentin, J. D. (2009). Interaction between TAK1-TAB1-TAB2 and RCAN1-calcineurin defines a signalling nodal control point. Nat. Cell Biol. 11, 154-161. doi: 10.1038/ncb1823

Martin, M. U., and Wesche, H. (2002). Summary and comparison of the signaling mechanisms of the Toll/interleukin-1 receptor family. Biochim. Biophys. Acta 1592, 265-280. doi: 10.1016/s0167-4889(02)00320-8

Moehle, M. S., Webber, P. J., Tse, T., Sukar, N., Standaert, D. G., DeSilva, T. M., et al. (2012). LRRK2 inhibition attenuates microglial inflammatory responses. J. Neurosci. 32, 1602-1611. doi: 10.1523/JNEUROSCI.5601-11.2012

Mogi, M., Harada, M., Narabayashi, H., Inagaki, H., Minami, M., and Nagatsu, T. (1996). Interleukin (IL)-1 $\beta$, IL-2, IL-4, IL-6 and transforming growth factor- $\alpha$ levels are elevated in ventricular cerebrospinal fluid in juvenile Parkinsonism and Parkinson's disease. Neurosci. Lett. 211, 13-16. doi: 10.1016/03043940(96)12706-3

Mogi, M., Harada, M., Riederer, P., Narabayashi, H., Fujita, K., and Nagatsu, T. (1994). Tumor necrosis factor- $\alpha$ (TNF- $\alpha)$ increases both in the brain and in the cerebrospinal fluid from parkinsonian patients. Neurosci. Lett. 165, 208-210. doi: 10.1016/0304-3940(94)90746-3

Moore, D. J., West, A. B., Dawson, V. L., and Dawson, T. M. (2005). Molecular pathophysiology of Parkinson's disease. Annu. Rev. Neurosci. 28, 57-87. doi: 10.1146/annurev.neuro.28.061604.135718

Park, J., Oh, Y., and Chung, K. C. (2009). Two key genes closely implicated with the neuropathological characteristics in down syndrome: DYRK1A and RCAN1. BMB Rep. 42, 6-15. doi: 10.5483/bmbrep.2009.42.1.006

Rothermel, B., Vega, R. B., Yang, J., Wu, H., Bassel-Duby, R., and Williams, R. S. (2000). A protein encoded within the Down syndrome critical region is 
enriched in striated muscles and inhibits calcineurin signaling. J. Biol. Chem. 275, 8719-8725. doi: 10.1074/jbc.275.12.8719

Russo, I., Berti, G., Plotegher, N., Bernardo, G., Filograna, R., Bubacco, L., et al. (2015). Leucine-rich repeat kinase 2 positively regulates inflammation and down-regulates NF- $\mathrm{KB}$ p50 signaling in cultured microglia cells. J. Neuroinflammation 12:230. doi: 10.1186/s12974-015-0449-7

Shin, N., Jeong, H., Kwon, J., Heo, H. Y., Kwon, J. J., Yun, H. J., et al. (2008). LRRK2 regulates synaptic vesicle endocytosis. Exp. Cell Res. 314, 2055-2065. doi: 10.1016/j.yexcr.2008.02.015

Sun, L., Deng, L., Ea, C. K., Xia, Z. P., and Chen, Z. J. (2004). The TRAF6 ubiquitin ligase and TAK1 kinase mediate IKK activation by BCL10 and MALT1 in T lymphocytes. Mol. Cell 14, 289-301. doi: 10.1016/s1097-2765(04)00236-9

Vega, R. B., Yang, J., Rothermel, B. A., Bassel-Duby, R., and Williams, R. S. (2002). Multiple domains of MCIP1 contribute to inhibition of calcineurin activity. J. Biol. Chem. 277, 30401-30407. doi: 10.1074/jbc.M200123200

Wang, R., Chen, Z., Fu, Y., Wei, X., Liao, J., Liu, X., et al. (2017). Plasma cystatin c and high-density lipoprotein are important biomarkers of Alzheimer's disease and vascular dementia: a cross-sectional study. Front. Aging Neurosci. 9:26. doi: $10.3389 /$ fnagi.2017.00026

Wang, W., Zhu, J. Z., Chang, K. T., and Min, K. T. (2012). DSCR1 interacts with FMRP and is required for spine morphogenesis and local protein synthesis. $E M B O \quad J . \quad 31,3655-3666$. doi: 10.1038/emboj. 2012.190

Wei, X., Gao, H., Zou, J., Liu, X., Chen, D., Liao, J., et al. (2016). Contra-directional coupling of Nur77 and Nurr1 in neurodegeneration: a novel mechanism for memantine-induced anti-inflammation and anti-mitochondrial impairment. Mol. Neurobiol. 53, 5876-5892. doi: 10.1007/s12035-015-9477-7

Windheim, M., Stafford, M., Peggie, M., and Cohen, P. (2008). Interleukin-1 (IL-1) induces the Lys63-linked polyubiquitination of IL-1 receptor-associated kinase 1 to facilitate NEMO binding and the activation of IкB- $\alpha$ kinase. Mol. Cell. Biol. 28, 1783-1791. doi: 10.1128/mcb.02380-06

Xu, Y. Q., Long, L., Yan, J. Q., Wei, L., Pan, M. Q., Gao, H. M., et al. (2013). Simvastatin induces neuroprotection in 6-OHDA-lesioned PC12 via the PI3K/AKT/caspase 3 pathway and anti-inflammatory responses. CNS Neurosci. Ther. 19, 170-177. doi: 10.1111/cns.12053
Xu, Y., Yan, J., Zhou, P., Li, J., Gao, H., Xia, Y., et al. (2012). Neurotransmitter receptors and cognitive dysfunction in Alzheimer's disease and Parkinson's disease. Prog. Neurobiol. 97, 1-13. doi: 10.1016/j.pneurobio.2012. 02.002

Yamazaki, K., Gohda, J., Kanayama, A., Miyamoto, Y., Sakurai, H., Yamamoto, M., et al. (2009). Two mechanistically and temporally distinct NF- $\mathrm{\kappa B}$ activation pathways in IL-1 signaling. Sci. Signal. 2:ra66. doi: 10.1126/scisignal.20 00387

Yu, Y., Ge, N., Xie, M., Sun, W., Burlingame, S., Pass, A. K., et al. (2008), Phosphorylation of Thr-178 and Thr-184 in the TAK1 T-loop is required for interleukin (IL)-1-mediated optimal NFKB and AP-1 activation as well as IL-6 gene expression. J. Biol. Chem. 283, 24497-24505. doi: 10.1074/jbc. M802825200

Yun, H. J., Kim, H., Ga, I., Oh, H., Ho, D. H., Kim, J., et al. (2015). An early endosome regulator, Rab5b, is an LRRK2 kinase substrate. J. Biochem. 157, 485-495. doi: 10.1093/jb/mvv005

Zanin, M. P., Mackenzie, K. D., Peiris, H., Pritchard, M. A., and Keating, D. J. (2013). RCAN1 regulates vesicle recycling and quantal release kinetics via effects on calcineurin activity. J. Neurochem. 124, 290-299. doi: 10.1111/jnc. 12086

Zhou, P., Weng, R., Chen, Z., Wang, R., Zou, J., Liu, X., et al. (2016). TLR4 signaling in $\mathrm{MPP}^{+}$-induced activation of BV-2 cells. Neural Plast. 2016:5076740. doi: $10.1155 / 2016 / 5076740$

Conflict of Interest Statement: The authors declare that the research was conducted in the absence of any commercial or financial relationships that could be construed as a potential conflict of interest.

Copyright (c) 2017 Han, Yoo, Sung, Chung, Um, Kim, Seol and Chung. This is an open-access article distributed under the terms of the Creative Commons Attribution License (CC BY). The use, distribution or reproduction in other forums is permitted, provided the original author(s) or licensor are credited and that the original publication in this journal is cited, in accordance with accepted academic practice. No use, distribution or reproduction is permitted which does not comply with these terms. 